\title{
Affective Responses to Image Color Combinations
}

\author{
Jan J. Koenderink ${ }^{1,2,3, *}$, Doris I. Braun ${ }^{1}$ and Andrea J. van Doorn ${ }^{1,2}$ \\ ${ }^{1}$ Abteilung Allgemeine Psychologie and Center for Mind, Brain and Behavior, Justus Liebig \\ University, Otto-Behaghel-Str. 10F, 35394 Giessen, Germany \\ ${ }^{2}$ Experimental Psychology, Helmholtz Institute, Utrecht University, Heidelberglaan 1, 3584 \\ CS Utrecht, The Netherlands \\ ${ }^{3}$ Lab voor Experimentele Psychologie, KU Leuven, Tiensestr 102, Bus 3711, 3000 Leuven, \\ Belgium
}

Received 17 August 2020; accepted 27 September 2021

\begin{abstract}
Responses to colored patterns were collected for a group of 60 naive participants. We explicitly aimed at affective responses, rather than aesthetic judgments, so this is not 'color harmony' proper. Patterns were mainly spatially highly structured compositions, the color palettes reminiscent of what is found in generic 'colorist' art. Color combinations systematically cover mono-, di-, and trichromatic chromatic chords, whereas there was always an additional achromatic component. This sets the research apart from the bulk of the mainstream literature on 'color harmony.' Various ways of analysis are compared. Clustering methods reveal that the responses are highly structured through the teal-orange (cool-warm) dimension. Clustering reveals a large group of mutually concordant participants and various small, idiosyncratic groups. When the data is coarse-grained, retaining only a limited redblue-yellow palette, the group as a whole appears quite concordant. It is evident that responses are systematic, thus the notion of a universal affective response to color combinations gains some credibility. The precise affective responses are specific because constrained by the seven categories used in the experiment. Thus, the systematic structure is perhaps to be understood as the generic result. We discuss tangencies with various traits found with 'colorist' art styles.
\end{abstract}

\section{Keywords}

Color, composition, affective response

\footnotetext{
* To whom correspondence should be addressed. E-mail: koenderinkjan@gmail.com 


\section{Introduction}

Why yet another study on 'color harmony' (as this paper will be categorized by the field - Burchett, 2002; Granger, 1955; Granville, 1987; Heddell, 1988; Moon and Spencer, 1944a, b, c; Pope, 1944) if there is already such a mass of existing literature from over the centuries? The point is that 'color harmony' is not a well-defined concept and indeed is rarely defined, except operationally, on a per study basis. This ambiguity is apparent from the variety of criteria and stimuli. So any nontrivial study that deviates from the trodden path has potential implications for the ontology of the matter.

The artist is unlikely to be impressed by the conventional stimuli used in the sciences (Fig. 1). Indeed, if the inquiry into affects of colors or color combinations is kindled by an interest in artistic problems, then these issues are serious ones.

An æsthetic opinion (say 'beautiful' or 'ugly') is simply too diffuse or vague. "Beauty is in the eye of the beholder" (see Note 1 - Hethorn, 2005; Hume, 1910 [orig. 1757]; Nietzsche, 1988 [orig. 1873]; Stone, 2004). One may very well value a piece of art because of its ugliness, either the object itself, or its nominal 'subject.' For an art collector 'beauty' is generally not the best reason to buy a work of art. One is likely to end up with an embarrassment a year later, when the beauty already starts to look bland or cheap. Moreover, there are many other dimensions than beauty. Even 'beauty' itself (whatever it might be) is certainly multidimensional (Zangwill, 2003).

It may well be that certain stimuli are just perfect for psychophysical purposes, but too boring to justify even a cursory look in real life. Unfortunately, most of the literature uses stimuli like that (Fig. 1). It is quite evident that responses to color combinations are highly dependent on both the structural and semantic make-up of the stimuli. This is also clear from the various crossmodal effects associated with color (Chen et al., 2016; Kim, 2013). Thus, stimuli can be too simple (say a split rectangular field filled with two uniform colors), but just as well too complicated (say random color photographs downloaded from the Internet; Solli and Lenz, 2011). Both extremes are perhaps to be avoided if one's aim is artistic relevance.
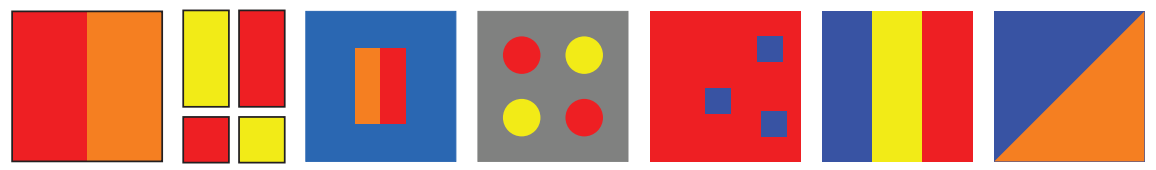

Figure 1. Typical stimuli harvested from the literature on 'color harmony.' These all have specific advantages and disadvantages for psychophysical or experimental phenomenological research. There is amply enough material for a voluminous review paper here. The stimuli used in this paper are categorically different. 
In judging a color scheme a visual artist will put a picture upside down, look through the eyelashes, or paint a scheme (Fig. 2). That is to say, anything that will yield a nontrivial abstract pattern can be judged on the basis of coloring. This removes the semantic annotations, but is quite different from using a color samples book from a paint manufacturer (for instance, Pantone, 2020).

These are reasons why most of the literature on color harmony is of little interest to the artist. It is perhaps useful as formula to architects and interior designers (Rijgersberg, 1938), or fashion designers (Stone, 2004). It allows them to discuss color schemes with potential customers before investing in actual (costly!) construction or manufacture.

From a scientific perspective, it is very clear that many papers published over the decades have very distinct objectives and that results are mutually noncommensurable. The main problem with that is that such incommensurable results will be headed under the same keywords, whereas mainly quantitative data are compared. It is a bit like monetary value that renders any incompatible commodities comparable. What really counts are criteria and especially stimuli. Data variation due to stimulus selectivity is likely to far exceed variability within paradigms. This is a very serious problem when not recognized for what it is (Yarkoni, 2020). Unfortunately, it is generally ignored.

We present an unpretentious evaluation of a small set of affective responses to a fairly large number of color combinations presented as patterns that are 'interesting to look at.' That the structure of the stimulus is of major importance is another case of Fechner's 'Associationsprincip' (Fechner, 1866; Ortlieb et al., 2020).

Indeed, various of our stimuli, when suitably framed and hung on the wall, would to many look better than the wall behind them (Note 2). That is the absolute minimum a painter has to achieve in order to make a living. This explains why our title does not include the term 'color harmony.'

It stands to reason that we construct stimuli in such a way that they are reproducible and can be simply parameterized (Supplementary Sections S2 through S6 (Note 3)). When the formal, algorithmic structure is unknown, such stimuli appear nontrivial and well-structured, yet at the same time fully unpredictable. In a sense 'fully structured' but 'unpredictable' is the aim of the arts. It is loosely related to concepts as 'Prägnanz' (Wertheimer, 1924) and George David Birkhoff's (1884-1944) formal notions (Birkhoff, 1933). This effectively combats potential boredom, but the strict parameterization distinguishes the stimuli from the many examples to be found in technical books on the art of painting. Arbitrarily many instances for any given set of parameters can be generated. These look all 'of a kind,' although different at closer scrutiny.

It will be clear from our discussion that there is no way to base experiments on hypotheses that might be simply falsified. Such methods have rarely been 


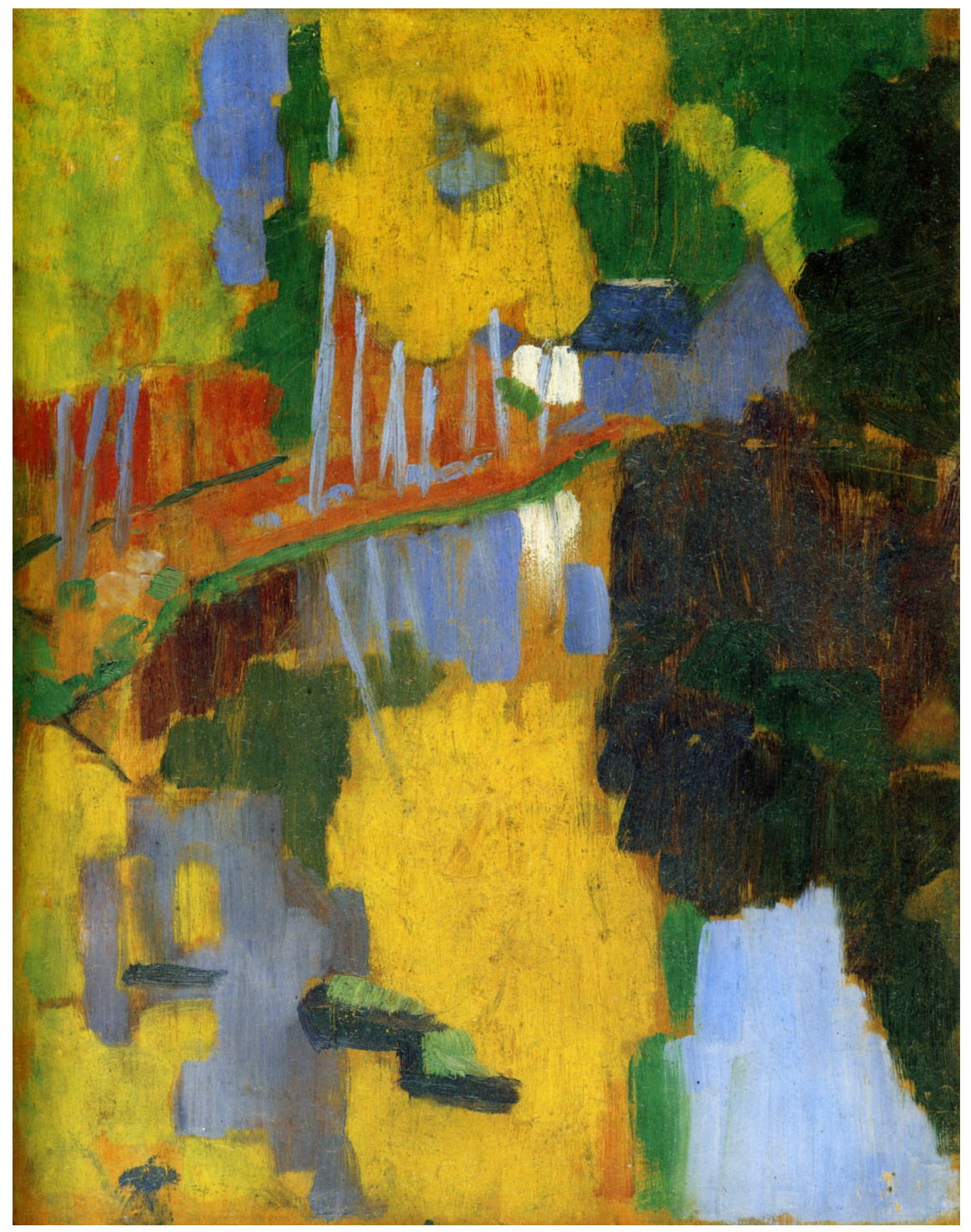

Figure 2. Paul Sérusier (1864-1927), Le Talisman (1888, under guidance of Paul Gauguin). This was the key image to the Nabis (1888 until 1900), hence the title (original title The Bois d'Amour at Pont Aven). Art historically, it represents a very early example of almost completely chromatic painting (KWYBR). It looks very similar when put upside down, although the compositional balance varies with the orientation. Sérusier painted it only on the cover of a cigar box. 
used in the relevant literature, the one striking example (but narrowly focused) being Moon and Spencer's (1944b) test of the theory of the mathematician Birkhoff (1933; Note 4). The most productive approach is likely to be frankly explorative; this implies experimental phenomenology (Albertazzi, 2013) and exploratory data analysis (Tukey, 1977) instead of the conventional confirmative methods.

Because of the limited scope, it would a priori be preposterous to expect to be able to draw generic conclusions from an experiment like this. As it turns out, we feel entitled to make some interesting connections with both conventional and historical artistic lore that appear to be novel. There is at least some potential academic interest.

\section{Color Preferences: Likes and Dislikes}

Before starting on the actual experiment, all participants were informally asked to mention their most preferred and least preferred colors, using any color names of their fancy. Although not a formal experiment we collected such data because it might conceivably aid in the interpretation of the formal data (Note 5). In earlier work (Albertazzi et al., 2015) we found that such data (which is easy to collect in the margin of the actual experiment) can be quite useful. Moreover, it puts the participants in the appropriate situational awareness.

With 60 participants we thus collected two sets (likes and dislikes) of 60 color names. From the total of 120, after discounting multiple mentions, we end up with 47 distinct names. These include such names as 'angry violet,' 'Flieder' (German for lilac), 'petrol,' 'khaki' and 'reseda green,' although the bulk are regular names (both in English and German) in common use (Supplementary Section S7). We used lists of color names regularly used by designers to convert all names to RGB coordinates. Then we converted each color to the nearest representative in a standard list, obtained by ranging the individual RGB coordinates over the values $\{0,1 / 2,1\}$ (that is $3^{3}=27$ in total). We find that participants had selected from 19 (either like or dislike) out of the standard list (Note 6).

The 19 colors include the 11 colors 'that will never be confused' (white, gray, black, red, green, yellow, blue, pink, brown, orange, purple) proposed by Boynton (1989). Green, yellow and blue account for 50\% of the mentions, adding purple, pink, brown, white, deep blue and red raises the fraction to $90 \%$, again adding cyan, pale yellow, gray, black, orange and light purple raises it to $99 \%$ (Fig. 3).

Blue and (to a lesser extent) green are the most preferred colors. When blue is the most preferred color, dislikes are green, red and purple. When green is the most preferred color, we find a dislike for red. 


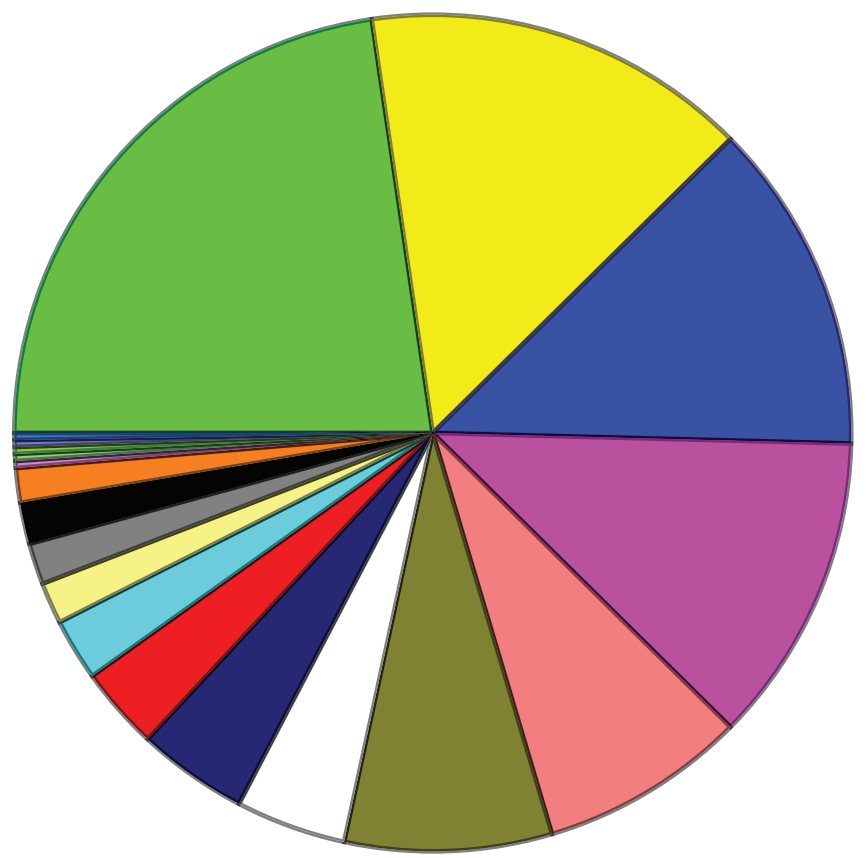

Figure 3. Colors used by the participants, mapped on the standard set. The pie chart gives a visual impression of the frequency of occurrence. (Note that this graph pools likes and dislikes.)

We summarized the data by a voting followed by a selection. The voting procedure involved computing the number of likes minus the number of dislikes for each of the colors. The selection involved ignoring cases with total vote $-1,0$ or 1 , thus keeping only cases with two or more consensus pros or cons. Results are visualized as pairs of pie charts, one for likes, one for dislikes (Fig. 4).

This procedure was repeated for partial data, relating to either female or male, or to either youngsters or elderly. For the ages we set the thresholds at the median age, which is 29 years (Fig. 5).

In order to judge whether there exist gender or age differences we compute a like-vector for each group. The like-vector is a list of the number of mentions for each of the list of 47 used colors. Two of such like-vectors can be compared in various way. We used the Ruzicka Similarity (Supplementary Section S6).

Although one readily computes similarities between groups (Fig. 6), it is not immediately obvious how to interpret these. A simple way to do it is to use resampling from the full group (Efron, 1981). For instance, when comparing genders we randomly select groups of the appropriate cardinality ( 37 for the females, 23 for the males) from the overall data (cardinality 60) and compute the similarity. We repeat this 10,000 times, which leads to an almost perfectly normal distribution. We use the mean and variance of this resampling 


\section{Overall}
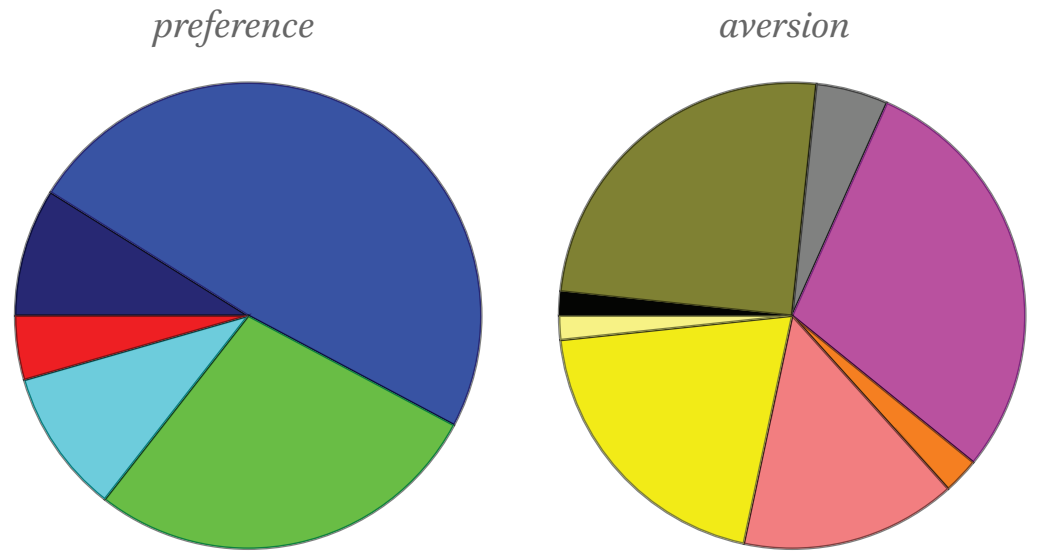

Figure 4. Pie charts of likes/dislikes for the whole group of participants.

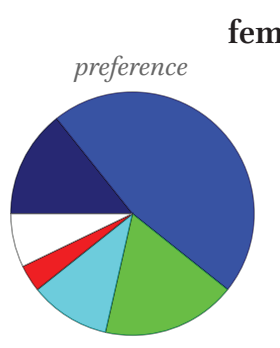

females

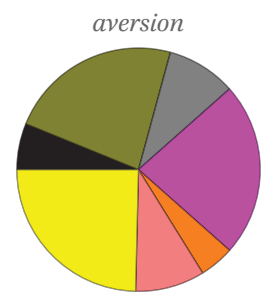

youngsters
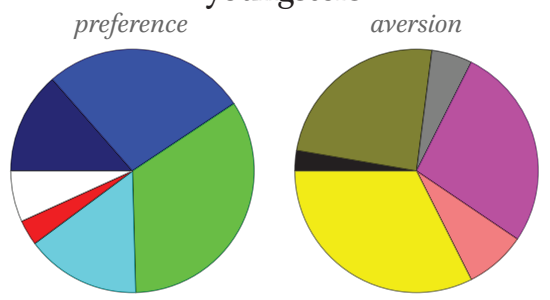
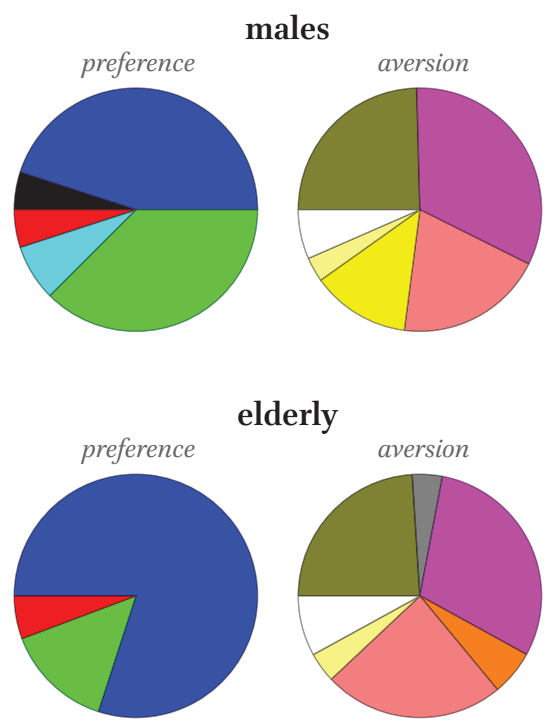

Figure 5. Gender and age preferences.

distribution to judge the actual intergroup similarities. We find an evident gender effect ( $p$-value 0.004) and also an evident age effect ( $p$-value 0.00002). Thus, both gender and age affect color preference. Repeating this for the dislikes, we arrive at a similar conclusion.

Such findings are, of course, hardly new (Note 7). 


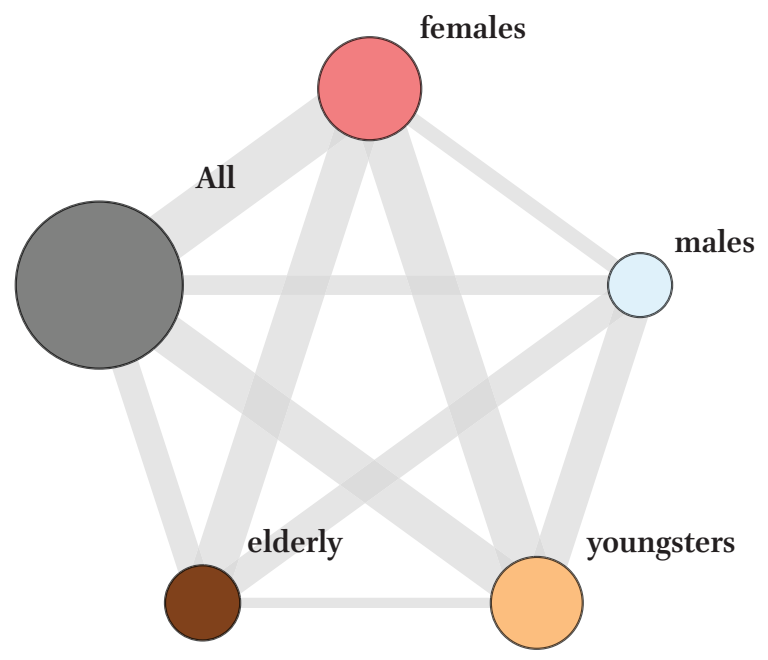

Figure 6. A graph of the similarity relations in preferences of subgoups. The vertex size indicates (sub-)group size, the thickness of the edges reflects similarity. Notice that the similarity between genders and that between age-groups is relatively low. This perception is validated by formal statistics.

\section{Participants}

Participants were students, scientific staff and administrative staff of the Giessen Department of Experimental Psychology and a few volunteers from the environment. Most had no particular affinity to or personal experience with visual art or design, but a few (less than 10\%) had. Some had earlier experience with experimental visual psychophysics, although not connected with the present topic. All had generic trichromatic vision.

There were 60 participants in total, 37 female, 23 male. Ages ranged from 17 to 77 years. The quartiles are $25(25 \%), 29(50 \%)$ and $38(75 \%)$. We have no record that details specific artistic interest/experience for all participants. Throughout the paper we use a binary distinction 'youngsters' and 'elderly,' where we set the boundary at the median. The overlaps between gender and age group are given by the Jaccard indices (Supplementary Section S6):

\begin{tabular}{lcc}
\hline & Youngsters & Elderly \\
\hline Female & 0.43 & 0.33 \\
Male & 0.27 & 0.28 \\
\hline
\end{tabular}


All participants were queried for their color preferences (limited to one most liked, one most disliked, otherwise free) before starting on the formal experiment. After the session we listened to an informal report of their impressions. Little was done with that, but such reports may yield ideas concerning interfaces and so forth.

\section{Design of Stimuli}

Experiments like this are nominally 'color harmony studies.' These include a large variety, and the many instances are ontologically distinct. Stimulus patterns are very diverse, they tend to be referred to by color name tuples. Lack of phenomenological discrimination leads to confusions and misconceptions. Mere nominal accounts, as common when literature is being quoted, fail to specify the meaning of the experiment, or the appearance of the stimuli.

We refer to stimulus patterns by color name tuples (say YG for some pattern containing yellow and green hues) in accordance to conventional use. This is explained in subsection 4.1. Color Tuples. But the actual stimulus patterns are of major importance and are likely to influence the results. Patterns are explained in subsection 4.2 Stimulus Patterns and Supplementary Sections S2 through S5.

\subsection{Color Tuples}

Generic observers discriminate at least a million colors (Note 8). Therefore the number of 'color combinations' is virtually infinite. The first design choice involves dealing with this cornucopia. Coarse-graining is natural because there do not exist more than about a dozen object colors that "will never be confused" (Boynton, 1989).

From a set of $N$ colors (here we count chromatic colors only, thus leave out white and black) one may obtain $\left(\left(\begin{array}{l}N \\ 2\end{array}\right)\right)$ distinct pairs and $\left(\left(\begin{array}{l}N \\ 3\end{array}\right)\right)$ triples, a total of $\frac{1}{6} N\left(N^{2}-1\right)$ combinations. Here we limit the subsets to those of cardinal-

ity 3 , for the total number of nonempty subsets is much larger $\left(2^{N}-1\right)$. For Boynton's set of 11 colors that implies 220 combinations, whereas there are 2,047 distinct subsets. Over 200 is a lot, especially for an experiment, but it is far less than the combinations for a set of $10^{6}$ items (Note 9).

We aim at not more than a few dozen combinations in order to keep the experiment within reasonable bounds. The problem is to select combinations in a principled way. This implies leaving out certain combinations, which goes at the cost of generality. 
We base the choice on the 'color circle' obtained from the subsets of the natural 'parts of white' (Koenderink, 2010) red (R), green (G) and blue (B). That leads to the set $\{\mathrm{K}, \mathrm{R}, \mathrm{G}, \mathrm{B}, \mathrm{C}, \mathrm{M}, \mathrm{Y}, \mathrm{W}\}$ of cardinality 8 . This includes cyan $(\mathrm{C})$, magenta $(\mathrm{M})$ and yellow $(\mathrm{Y})$, as well as the achromatic colors black (K) and white (W.) The chromatic colors are naturally ordered in the periodic sequence RYGCBM (and R again). There are $2^{8}-1=255$ nonempty subsets (Note 10). Thus, additional constraints are required.

This is necessarily a somewhat arbitrary choice. These are desiderata:

- Each subset should contain the achromatic colors. The reason is that purely chromatic choices make tonal painting very hard. Such palettes are mainly of interest in purely abstract compositions. Notice that this constraint is rarely recognized in the literature. In fact, the achromatic colors are conventionally ignored;

- The number of pure colors in the subset is limited to three. The reason is that for higher cardinalities one obtains a 'cacochromy.' Essentially all effective painter's palettes are 'limited.' Some painters (like famously Anders Zorn; Note 11) were often copied, or emulated, because of their palettes.

This fits in with the classical color harmonies, the 'triadic chords' being the most complex.

Thus, one ends up with the 42 subsets illustrated in Fig. 7. We have 1 achromatic combination, 6 monochromatic combinations, 15 dichromatic combinations and 20 trichromatic combinations. This is likely to be 'overkill,' as the number of generally acknowledged harmonious schemes is much less.

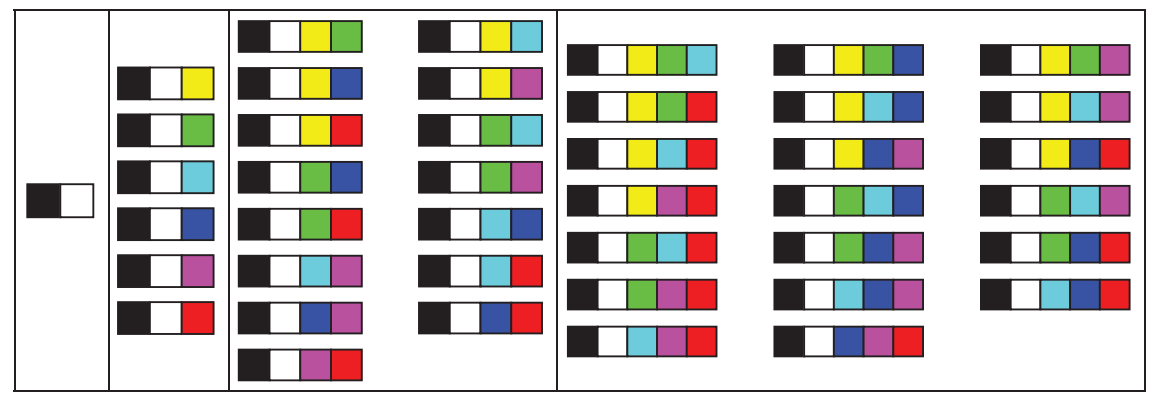

Figure 7. All forty-two constrained subsets, collected on the basis of cardinality. 


\subsection{Stimulus Patterns}

'Color harmony' (as generally used) is really a misnomer, because the affective value of colored patterns cannot be understood if the spatial pattern is ignored. Moreover, the qualities of pattern (Ostwald, 1922) are even more elusive than those of color. We use two basic patterns of a textural type (subsection Texture Patterns, and subsection Mosaic Patterns below) and a third pattern (subsection Foreground/Background Patterns below) that involves 'foreground' and 'background' (Note 12). Thus, we look no further than the tip of the iceberg.

One aspect in which our choice differs essentially from the mainstream conventions is that our stimuli are not immediately recognized as trivial or boring. Indeed, most participants told us that they enjoyed the task because the stimuli were so pleasant to look at. The task was at no time boring because of looking trivial.

Is this relevant? We tend to think so, but we will not defend this notion against all comers. There might well be an artist-scientist dichotomy here.

\subsubsection{Presenting the Subsets as Spatial Patterns}

In presenting a combination (or subset) one needs to decide on some spatial pattern. For our purposes we require spatial patterns in which all members of the subset are represented equally in some appropriate statistical sense. This rules out various common methods in which relative size, order, or left/right position tend to complicate the issue. One solution is a uniform statistical presentation. Of course, this can be implemented in infinitely many ways.

We use two instances, 'textures,' and 'mosaics.'

Texture Patterns. We generate gaussian random fields of $512 \times 512-$ pixels with power spectrum falling off with an exponent -1.2 . We then limit the pixel values to a finite range by applying an error function nonlinear compression in such a way that the histogram of the gray levels becomes uniform on $[0,1]$ (Supplementary Sections S2 and S3).

Additive combinations of such fields can be used to present a color set of cardinality $N \geq 1$. We simply generate $N$ independent instances. Then we use the pixel values as weights in a linear blend (Supplementary Section S2) of the colors.

It should be understood that this method leads to pixel histograms that are fairly concentrated, even when the weights (before normalizing by dividing by the total) are drawn from a uniform distribution on $[0,1]$. This has the advantage that the images look more 'unified,' they do not tend to 'fall apart visually' (Fig. 8; Note 13; Supplementary Sections S2 and S3).

Figure 9 shows examples of an achromatic pattern, a monochromatic pattern (red), a dichromatic pattern (red and green) and a trichromatic pattern (red, green and blue). All these include black and white. 

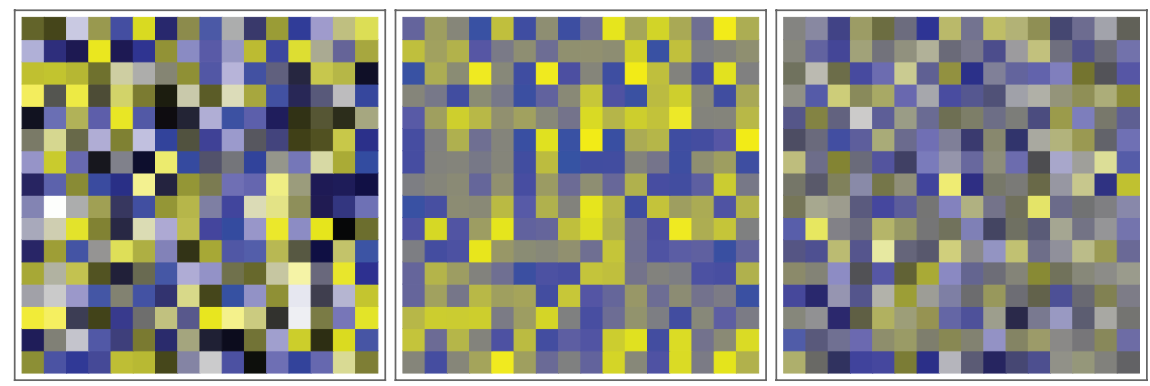

Figure 8. An example involving yellow-blue. At left pixels are defined as RGBColor $[\alpha, \alpha, \beta]$, where $\alpha, \beta$ are drawn from a uniform distribution of the unit interval. At center we draw two such uniform variates $\{\alpha, \beta\}$, normalize such that $\left\{\alpha^{\prime}, \beta^{\prime}\right\}=\{\alpha, \beta\} /(\alpha+\beta)$ and define pixels as RGBColor $\left[\alpha^{\prime}, \alpha^{\prime}, \beta^{\prime}\right]$. Finally, at right, we draw four such uniform variates $\{\alpha, \beta, \gamma, \delta\}$, normalize such that $\left\{\alpha^{\prime}, \beta^{\prime}, \gamma^{\prime}, \delta^{\prime}\right\}=\{\alpha, \beta, \gamma, \delta\} /(\alpha+\beta+\gamma+\delta)$ and define pixels as RGBColor $\left[\beta^{\prime}+\gamma^{\prime}, \beta^{\prime}+\right.$ $\gamma^{\prime}, \beta^{\prime}+\delta^{\prime}$ ], that is an even blend of black (weight $\alpha^{\prime}$ ), white (weight $\beta^{\prime}$ ), yellow (weight $\gamma^{\prime}$ ) and blue (weight $\delta^{\prime}$ ). The latter blend is the one used in the texture patterns. The first method (left) is perhaps more typical for the mainstream literature. [The quantitative differences are best judged from histograms of RGB-coordinates (Supplementary Section S2), but the visual effect is immediately apparent from these samples.]
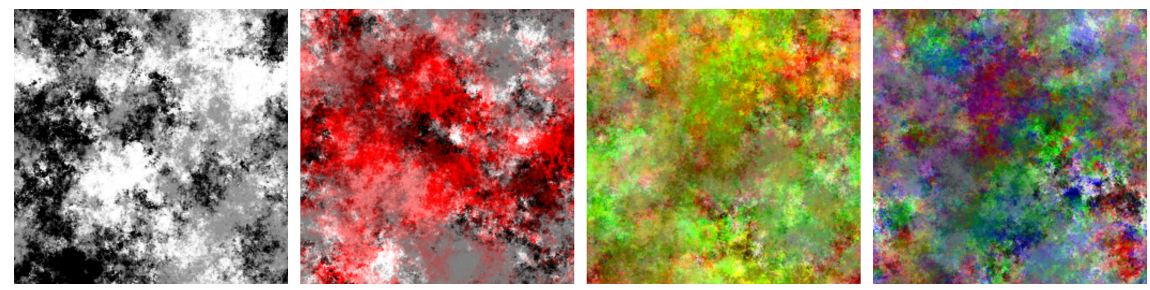

Figure 9. Examples of texture patterns. From left to right: an achromatic pattern, a monochromatic pattern (red), a dichromatic pattern (red and green) and a trichromatic pattern (red, green and blue). As explained (Fig. 8) all patterns include black and white. Such patterns are rather unlike typical patterns used in the literature (Fig. 1), which tend to be closer to a bi-partite or tri-partite field of uniform areas.

Mosaic Patterns. Voronoi patterns are random, discrete tilings, based on random, uniformly distributed points (Supplementary Section S4). Such tilings are useful because, like the noise images discussed in the previous section, they treat all colors in a subset equally in terms of the spatial distribution. This is conceptually important, since the spatial distribution - if not treating all colors equally - is likely to introduce a bias due to spatial position or sequence. For instance, in the classical landscape one has horizontal bands ranging from brown over green to blue from bottom to top (Koenderink et al., 2015).

With a tiling method one is completely free in the choice of sampling method, which is an advantage as compared to the noise patterns that require 
one to use the weighted means sampling. Here we selected a sampling method that comes somewhat closer to conventional choices in discussions on color harmony.

The main deviation from mainstream usage is that we tone down pure hue in the interest of a uniformity of pattern. We seek to approach presentations with some affinity to artistic methods, so we attempt to avoid the cacochromy that results from using samples of pure hues in some kind of fine-grained texture. This is easily done by introducing graying, that is, by admitting tints and shades, even grayed down hues, next to the pure colors. Such a toning down avoids cacochromia and serves to visually pull the pattern together. It is a palette not unlike Arnold Böcklin's, who actually applied the Ostwald ideas (Ostwald, 1917a, b, 1919) avant la lettre in freely mixing white and black with pure hues (Note 14).

The toning down can be achieved in many ways. We use a sampling of the classical Ostwald triangle using the weighted means method with uniformly distributed weights on the vertices (Supplementary Section S2).

Of course, one has an Ostwald triangle for any hue. In this study we use the cardinal hues red, yellow, green, cyan, blue and magenta. Their Ostwald triangles foliate the RGB cube in a simple manner (Note 15). The difference with the texture patterns is that they present hues from the full color circle, where we have only the cardinal hues in the mosaics. Thus, orange may occur in the textures (it is between red and yellow), but not in the mosaics (for there is no cardinal hue between red and yellow).

In order to implement the presentation of a subset, we simply sample uniformly from the Ostwald triangles of the members of the subset, using a uniform distribution. The resulting stimuli (example Fig. 10) 'look good' to most participants, although there is an overall preference for the textures.

Foreground/Background Patterns. In the 'foreground/background patterns' (Fig. 11) the 'background' is simply a texture image. The 'foreground' is a
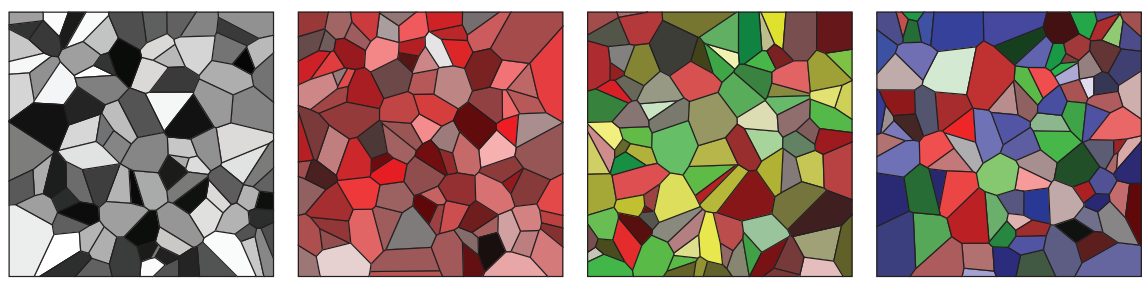

Figure 10. Examples of mosaic patterns. From left to right: an achromatic pattern, a monochromatic pattern (red), a dichromatic pattern (red and green) and a trichromatic pattern (red, green and blue). As explained (Fig. 8) all patterns include black and white. Like the textures, such patterns are also rather unlike typical patterns used in the literature (Fig. 1). They also appear as very different from the texture patterns (Fig. 9), although the nominal patterns (just a tuple of color names!) of Figs 9 and 10 are identical. 

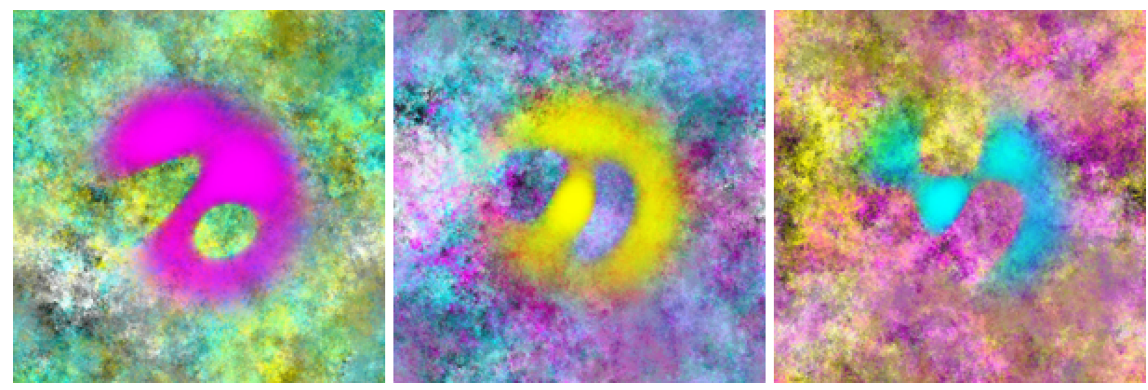

Figure 11. Some foreground/background examples. Notice that the foreground shape visually 'belongs to the picture' because of the lost-and-found edge quality. The reason is that we attempt to force observers to look at the pattern as a whole. (One never knows, but at least one can try.) From left to right: magenta foreground on yellow-cyan background, yellow foreground on magenta-cyan background, and cyan foreground on yellow-magenta background. Note all images involve the triple cyan-magenta-yellow. Yet how different they look! We use such examples to suggest that the nominal designation cyan-magenta-yellow is perhaps wanting in explanatory power. Picture sets like this almost enable 'instant psychophysics' (Richards, 1987).

random inkblot-like shape of a single color (Supplementary Section S5). The foreground is generated as a halftone image; we use the intensity as opacity for a uniform hue filling the whole image. Where the opacity is 0 one sees the background, when it is 1 one sees the foreground. The 'soft' edges serve to render the foreground as a true part of the image, not an arbitrary collage. Most instances indeed look like compositions that one might want to frame and hang on the wall. The participants were partial to them.

From a conceptual perspective, what counts is that the foreground although belonging to the picture - is clearly distinct from the background. This is a potential game-changer. The affective impact of colored patterns is not likely determined by mere nominal designations (color name tuples).

\section{Color Families and Conventional Color Chords}

The six-step color circle is presented in Fig. 12, top left. We use it to point out some conventional hue familes. The color circle has been oriented so as to naturally partition into two parts by the central vertical.

At the right side one has the warm hues, red and yellow (Fig. 12, top center), whereas at the left one has the cool hues cyan and blue (Fig. 12, top right). The central warm hue is orange, the central cool hue is teal. Teal-orange is the basic gradation in the visual arts (Fig. 13; Koenderink and van Doorn, 2021; Quiller, 1989; Note 16). It also comes out prominently in the experimental phenomenology of single colors (Albertazzi et al., 2015). 

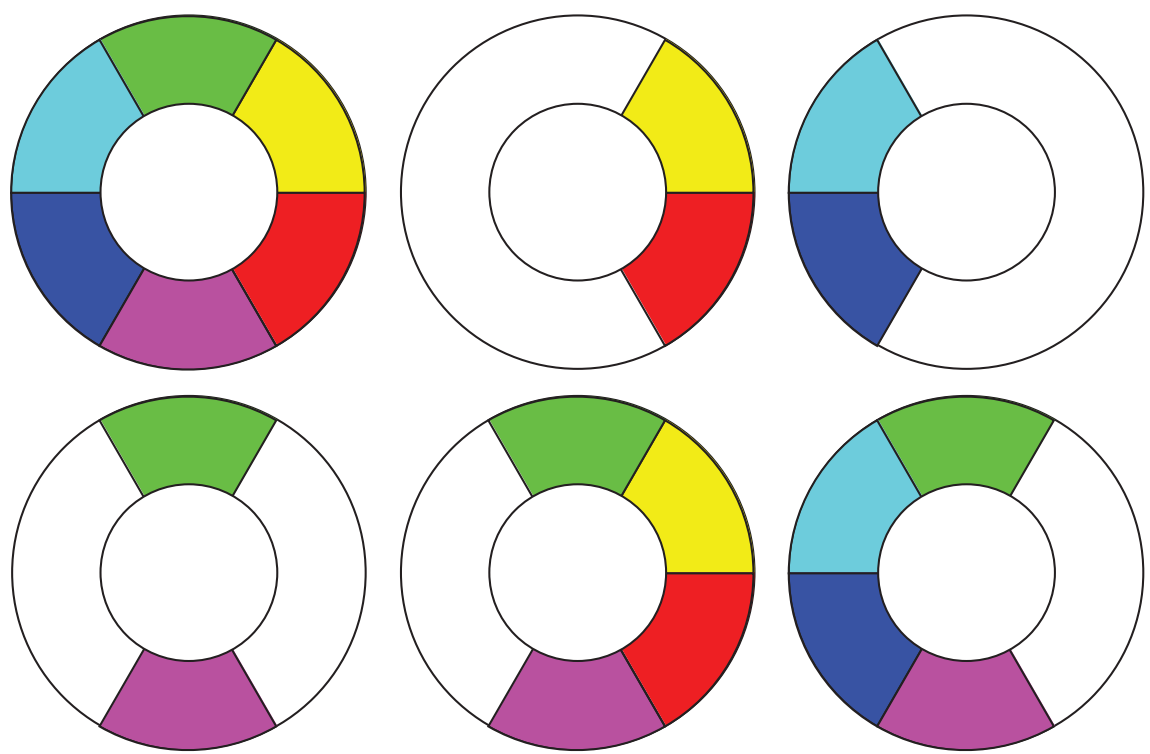

Figure 12. At top left the six-step color circle, at bottom left the neutral hues. At top center and right the pure warm and cool hues, at bottom center and right the augmented (with the neutral) warm and cool hues.

The hues on the vertical are green and magenta (Fig. 12, bottom left); they may be termed 'neutral' with respect to the basic warm-cool dichotomy. (Notice that 'neutral' is often used for gray tones, in this paper we will speak of 'achromatic' in case of the grays.)

In applications the neutrals may go either with the warms or with the cool, depending upon the composition. Thus, Fig. 12, bottom center, shows the augmented warm hues, whereas Fig. 12, bottom right, shows the augmented cool hues. This is very much like the case of 'middle gray,' which may go with the darks ('low-key' images) or lights ('high-key' images) as the case may be.

Thus, monochromatic combinations KWY or KWR are overall warm, KWC or KWB are overall cool, whereas KWG or KWM are referred to as 'neutral'. The dichromatic combinations KWGY and KWMR are both 'warm', KWGC and KWBM are both 'cool', whereas we will refer to KWRB as 'mixed' and KWGM as 'neutral'. This reflects common usage in art and design. In 'mixed' combinations one typically puts the cools in the background and the warms in the foreground, or vice versa (Note 17).

In artistic color harmony (Albers, 1963; Carpenter, 1915; Chevreul, 1855; Chijiiwa, 1987; Goethe, 1810; Kobayashi, 2010; Ostwald, 1918; Pawlik, 1969; Quiller, 1989; von Bezold, 1874) one often speaks of 'chords,' like in music (Rijgersberg, 1938). The simplest is, of course, the single tone. We refer to these as 'monochromatic tones' (Note 18). Monochromatic chords may be 


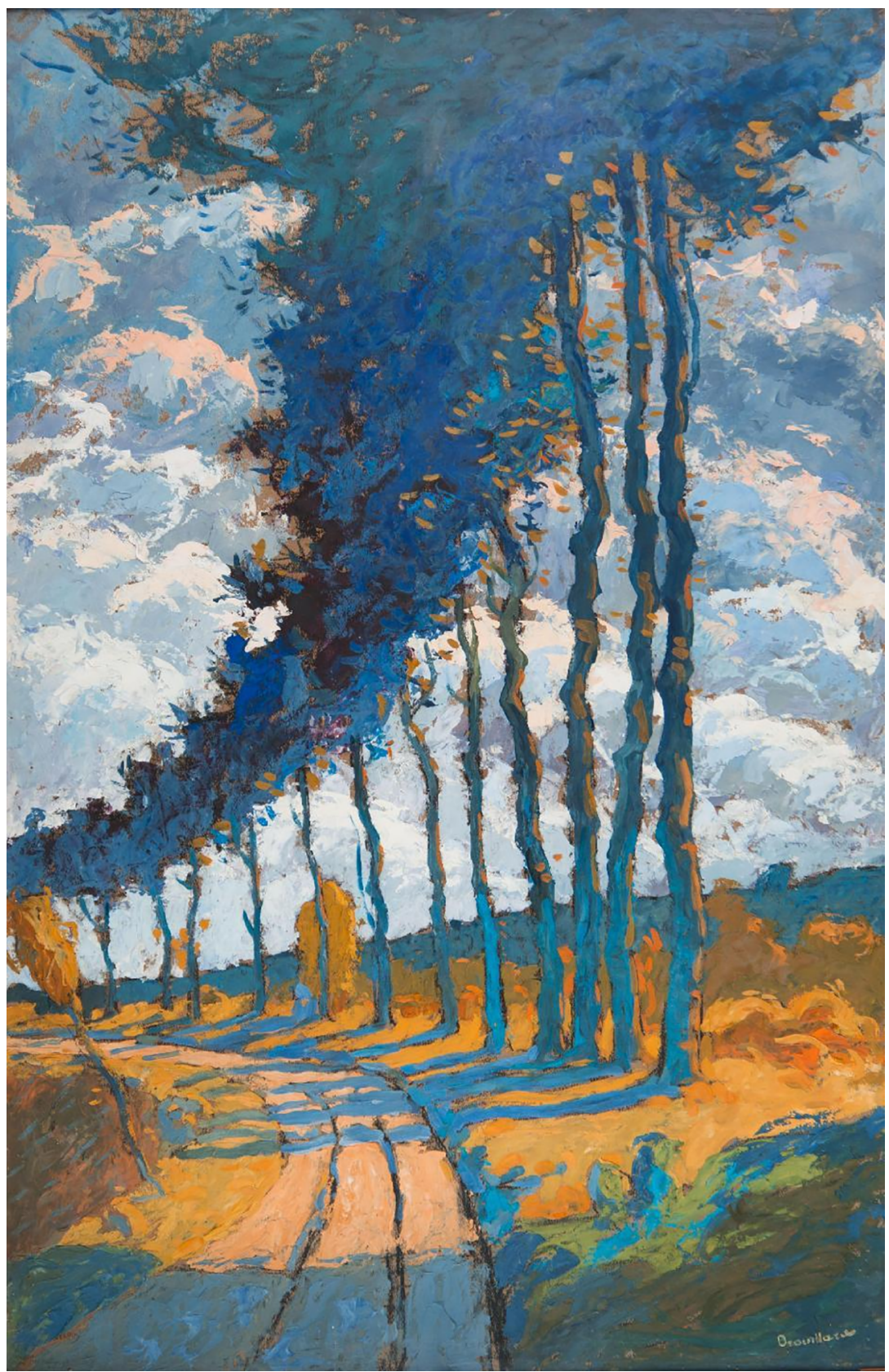

Figure 13. Eugène Brouillard (1870-1950), Arbres en Bordure d'un Chemin. Perhaps an extreme example (though common enough!) of the 'teal-and-orange' palette. It is certainly not 'natural' in the impressionist sense. Even such a minimalist palette is nevertheless 'complete.' This is where most of the chromatic action is. Other hues could provide 'accents,' it might work well enough in sufficiently small doses. 

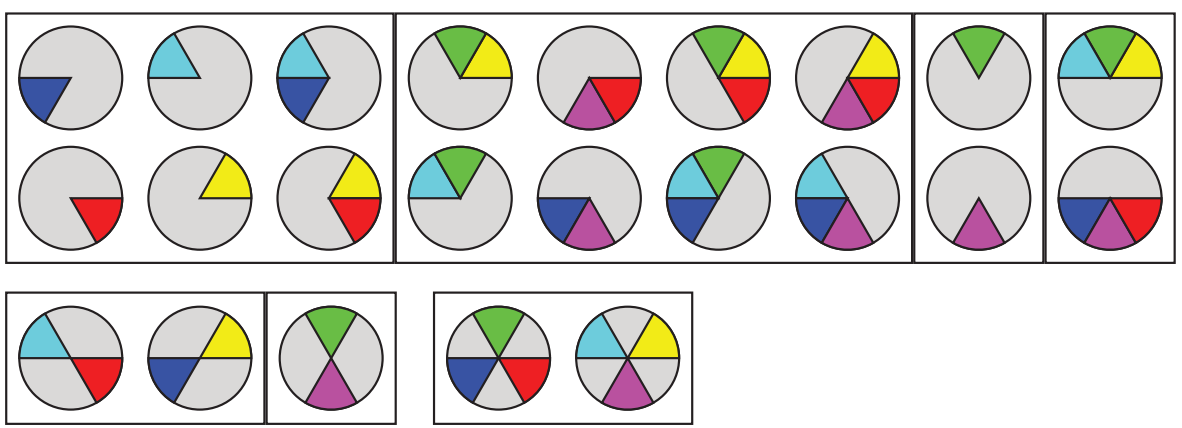

Figure 14. The various combinations used in the experiment. Here we do not show 18 'skew' combinations that do not naturally fit a conventional chord. In the boxes at top one has analogous schemes, from left to right: cool (top) or warm(bottom), augmented warm (top) or cool (bottom), neutral, and mixed, or perhaps 'augmented neutral.' Note mono-, di- and trichromatic instances. In the boxes at bottom, at left complementary pairs, either cool-warm (left), or neutral (right). At right the two trichromatic chords. There are 23 classical chords and 18 skew combinations, thus about fifty-fifty. When the achromatic pair is added, there are 42 combinations in total.

trivial and perhaps boring, but they are all 'harmonious.' Note we have six of these (Fig. 14 top).

Dichromatic combinations are of various types. There are ()$\left.^{(2)=4}\right)=15$ of them, but some are 'skew,' that is, do not correspond to a conventional chord. There are six dichromatic chords that are generally considered harmonious; most important of these are CB (teal) and RY (orange) (Koenderink and van Doorn, 2021). Other ones, like GY are often considered somewhat 'off-key.' Such adjacent pairs are generally denoted 'analogous color schemes' (Fig. 14 top left and center). These may be extended into broader ranges, like RYG, or MRY (two 'off' oranges). Analogous schemes cannot straddle the neutral axes, thus YGC would not count as such.

There are three dichromatic chords composed of complementary colors (Fig. 14 bottom left). Of these, RC and YB straddle the neural axis, whereas GM is different. In the art one member of the pair will usually be applied as an accent, whereas the other could be extended into an analogous range. Such 'analogous with complementary accent' schemes are the bread and butter of visual design.

Finally, except from the analogous combinations, there are two trichromatic chords (Fig. 14 bottom right), namely RGB and YCM. They are tricky to use, except when one or two of the components can be limited to a few image features that have an existence on the basis of figural (not purely chromatic) structure. They find use in architecture and design. 
Of course, the classical chromatic chords do not play a role as stimulus parameters in our experiment. We have mentioned them here because they play a role in the selection of chromatic combinations for stimulus material. As discussed earlier, this is forced on us because the sheer number of combinations easily reaches magnitudes that put them far outside the range of viable experiments.

We will return to the topic of chords after the formal analysis of the experimental results has been completed.

\section{Design of the Experiment}

We use a questionaire on the basis of seven five-point Likert scales. The limit of seven questions is constrained by the size of the display and the expected session times. We need to display the stimulus and the various scales on a single screen layout.

The bipolar categories used in the experiment are intentionally not aimed at aesthetic, but rather at affective judgments. We would expect them to be mutually correlated, but at least amount to four or five independent dimensions.

Note that the categories are listed in both English and German. That is also how they were announced on the display.

Here is the list:

1. soothing - irritating

2. weak - strong

3. dull - vivid

4. subdued - intrusive

5. calm - lively

6. blunt - piercing

7. gloomy - cheerful

$$
\begin{aligned}
& \text { beruhigend - irritierend } \\
& \text { schwach - stark } \\
& \text { dumpf - lebendig } \\
& \text { zurückhaltend - aufdringlich } \\
& \text { ruhig - lebhaft } \\
& \text { stumpf - stechend } \\
& \text { schwermütig - fröhlich }
\end{aligned}
$$

All participants were familiar with English, but many had German as mother tongue. One participant had Turkish as first language. In all cases we tried to make sure the categories were understood in the intended way.

In view of the difficulty of the task we judged a five-point scale to be sufficient. Although it is likely that participants will hesitate to use the extremes, this offers a way to avoid the 'neutral' response. We expect that mainly the polarity of excursions from the neutral response, no matter their magnitude, is likely to be informative. We do not share the conventional assumptions associated with Likert scales. This implies that the analysis has to be more basic. 


\subsection{Presentation and User Interface}

Stimulus and questionnaire were presented on the computer screen (screen grab in Fig. 15). The participant used the mouse to click one of the five levels of the Likert scale. Categories could be responded to in any sequence. Participants were free to change their judgment of any category at any time. When all categories had been responded to, the yellow square (see the screen grab) appeared and the participant could finalize the final set of responses to all categories. After clicking the yellow square the next stimulus was presented and the yellow square would disappear. When all stimuli had been responded to, the program would automatically exit.

Notice that the background, the interface elements and the texts are rendered in gray tones in order to reduce any possible interference with the task. (The yellow square only appears when all judgments have been indicated.) The stimulus is isolated through a broad, black frame.

We used Apple MacBooks with calibrated and linearized LCD displays (Supplementary Section S1) viewed binocularly at normal reading distance, using personal viewing aids when desired. The participants interacted with the interface via the mouse.

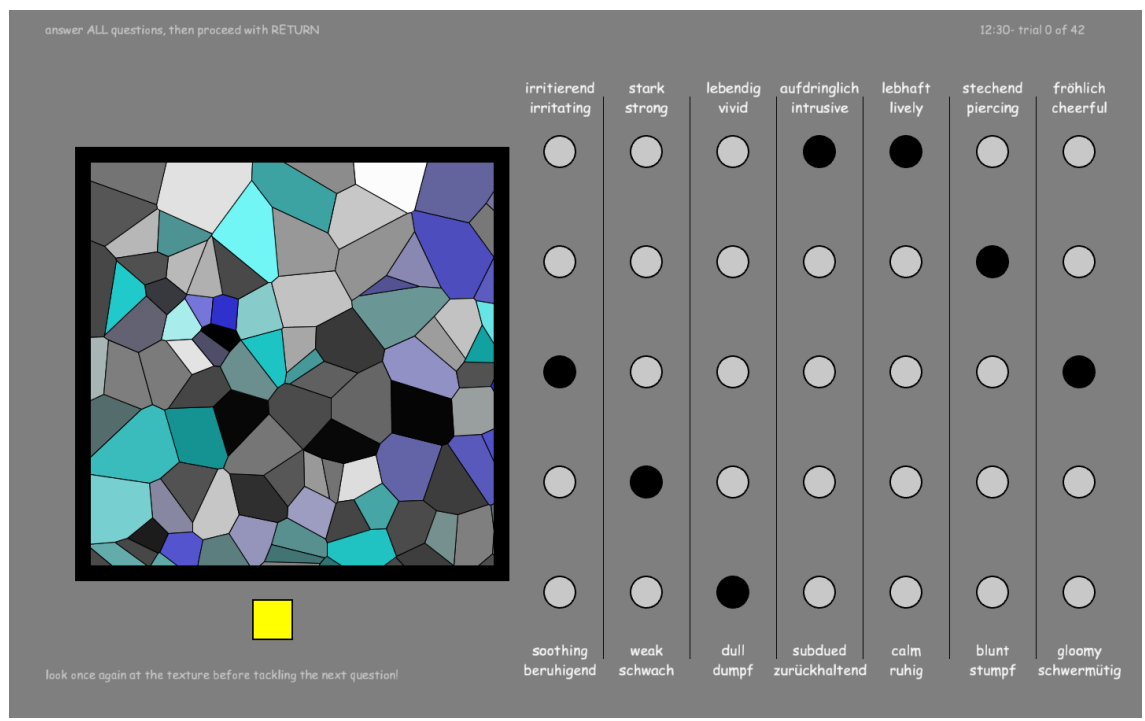

Figure 15. A screen grab of the experiment at a moment that all responses were completed, so the yellow square is presented. Clicking the yellow square will initiate the next trial. 


\section{The Raw Data}

The first analysis simply probes whether there is any structure in the data at all. After all, 'color harmony' is generally considered as hardly a suitable topic for scientific investigation. So it is certainly possible that the data would turn out to be completely random.

Moreover, it is a priori likely that people might be different in this respect. That De gustibus et coloribus non est disputandum is an ancient Roman adage. Its roots are not even known. It can hardly be complete nonsense. So we have to reckon with the existence of groups and singular outliers.

In this section we take an overall look at the data, with the intention to investigate whether a finer analysis might make sense.

\subsection{Use of the Likert Scale}

Do participants appear to use the Likert scale in the intended sense? One may attempt to find out by counting. In Fig. 16 we count the use of the scale steps over all participants, color combinations and response categories. We find that all participants use all scale levels. The neutral level and the step below are most common, extreme values are much less frequent. This is indeed what one likes to see.

In Fig. 17 left we consider the scale use for the individual categories. This looks fine too. There is an indication that categories might indeed be different. A dendrogram (Fig. 17 right) reveals this structure (Note 19). Apparently the calm-lively and dull-vivid dichotomies are quite similar and so are bluntpiercing and subdued-intrusive. Intuitively, the structure appears reasonable, or, at least, not wildly unlikely.

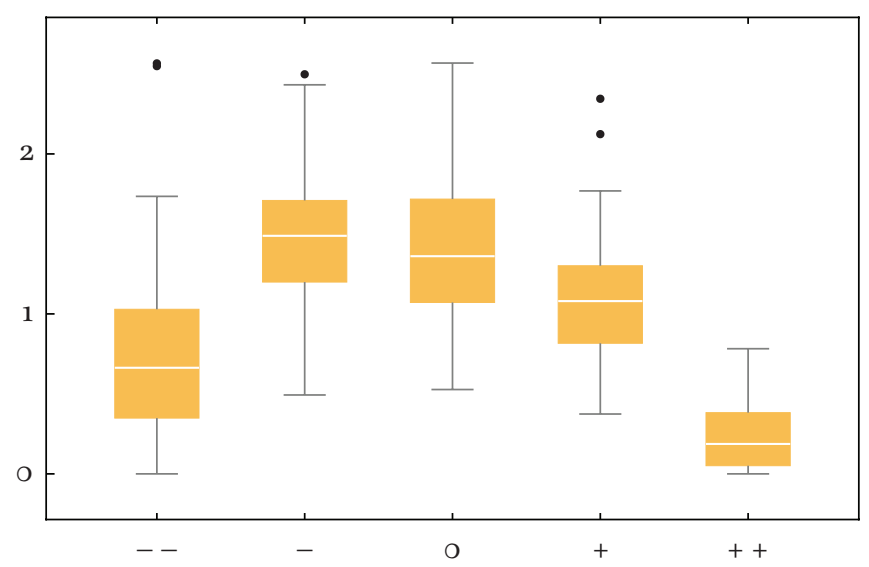

Figure 16. The overall use of the Likert scale. 

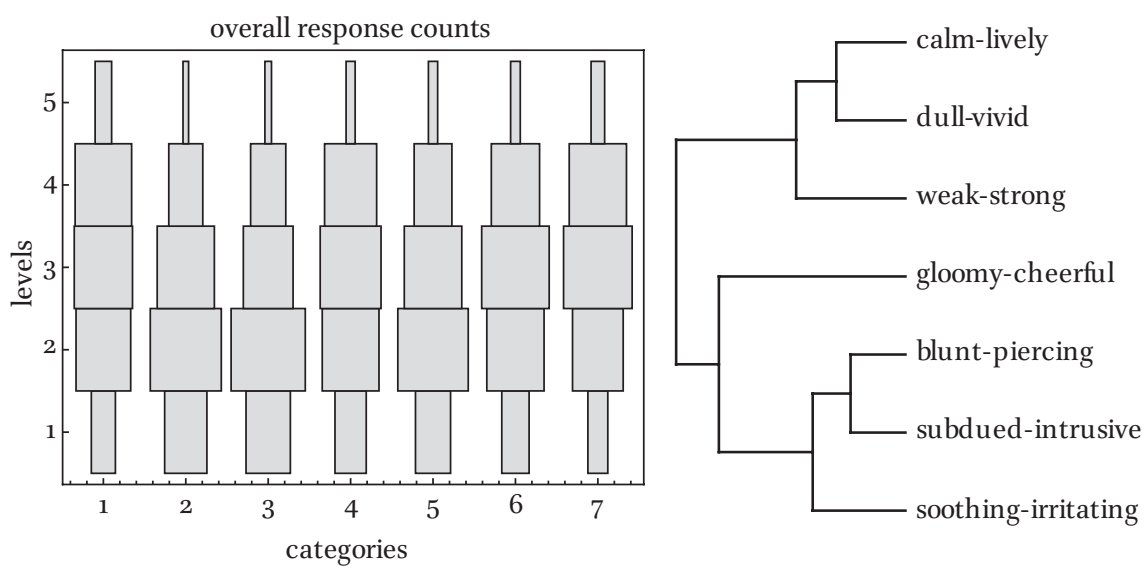

Figure 17. Left: Overall counts over all participants and color combinations show that there might be some variation in the seven categories. Right: A dendrogram reveals the nature of mutual clustering among the categories.

We find that the responses averaged over all participants are very nearly normally distributed with mean -0.1434 and standard deviation 0.248 (Note 20).

A very simple, assumptions-free, method to compare participants is to divide the participants randomly into two groups of equal size and compare these. As shown in Fig. 18 such groups are highly correlated (Note 21). Repeating the bisection a thousand times yields a median coefficient of variation 0.89 with an interquartile range of $0.88-0.90$. Apparently the participants have a definite overall concordance.

\subsection{Distinguishing Participant Groups}

As a next step we may compare pairs of participants over all color combinations and categories. A good overview considers the median of the correlation of a given participant with all others. The total range of values over all participants is $[-0.11,+0.61]$. Thus, there are participants that anti-correlate(!) with more than half of all others. This suggests strongly that one should attempt to identify one or more groups of mutually concordant members.

A simple start is to split at the median (correlation 0.476). This turns out to yield a group of half the participants that is indeed mutually concordant and very different from the remaining group (Fig. 19).

When attempting dendrograms, or clustering, one identifies this same concordant group, whereas the remainder falls apart in many much smaller groups. We decided to do the more detailed analysis both for the concordant group and 


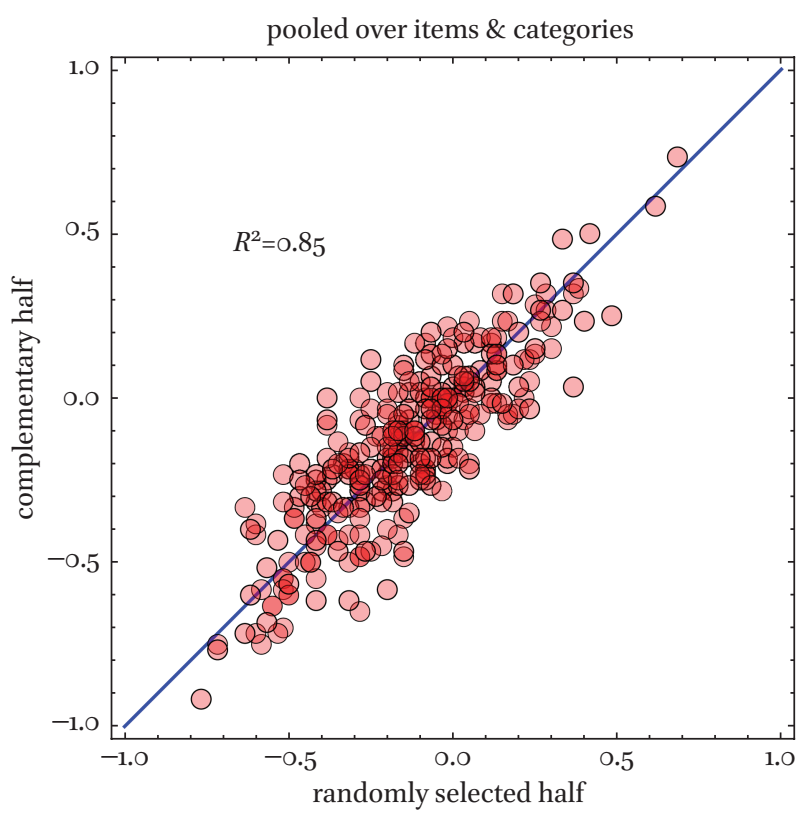

Figure 18. Randomly splitting the participants in two halves and comparing the groups on particular color combinations and categories reveals a fairly strong dependence. [Here 0.0 is the neutral level, \pm 1 a single step up or down in the Likert scale $(-2,+2)$.] This is just one random case, but we find that it is typical for any random partition. It indicates that the data is systematically structured.

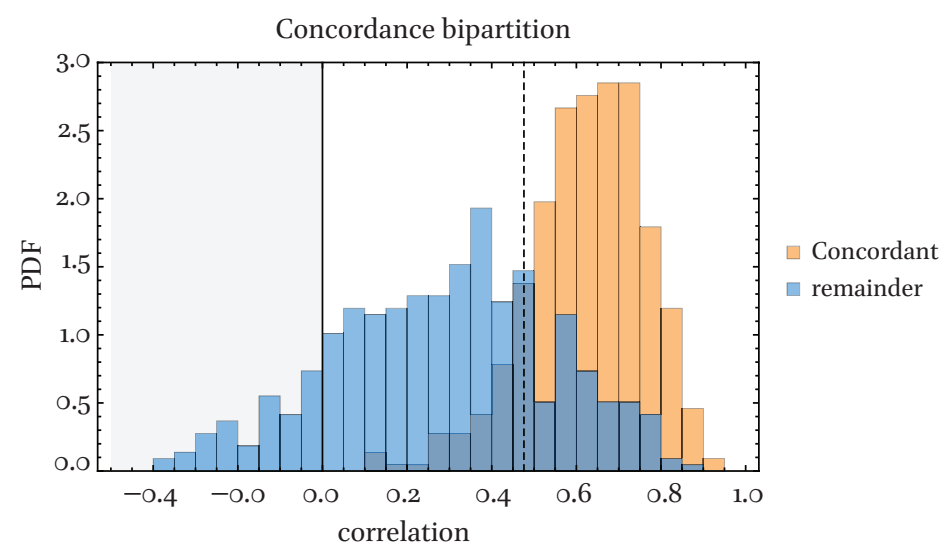

Figure 19. This is a histogram of pairwise correlations in two groups of participants, a 'concordant' group and the remainder. The 'concordant' group members are highly correlated among each other. The median is 0.63 , interquartile range $0.54-0.72$. Members of the remainder group are at best weakly correlated with the others. The median is 0.31 , interquartile range $0.10-0.46$. Thus, the 'concordant' and 'remainder' groups are really different and have only minimal overlap. It may not be an (in some sense) optimal bipartition, but it is certainly effective. 
for the full group. The former will be most informative, whereas the latter yields an indication of genericity.

The concordant group has 17 females, 13 males and 18 youngsters, 12 elderly. The matrix of overlap (Jaccard indices; Supplementary Section S6) is

\begin{tabular}{lcc}
\hline & youngsters & elderly \\
\hline female & 0.40 & 0.32 \\
male & 0.35 & 0.25 \\
\hline
\end{tabular}

We conclude that the composition of the concordant group is a fair reflection of the composition of the total group.

To find such a highly concordant group consisting of half the participants (Note 22) is an interesting result by itself. It shows that the saying De gustibus et coloribus non est disputandum may well make some sense, but that it does not universally hold. Apparently major groups of people mutually agree on many issues involving color combinations. There exists a systematic structure.

These findings suggest that a finer-grained analysis might make sense. In the detailed analysis we put some emphasis on the concordant group, although we also report on the full group.

\section{Analysis}

For each color combination we obtain a 'response profile' by averaging over participants of the concordant group and considering the distribution over categories. When we summarize such profiles through the three strongest category responses, we obtain a clustering. Many color combinations form a cluster by themselves, but some cluster together with others. In Fig. 20 we show the most important clusters.

A somewhat less coarse way to characterize such response profiles for the group of concordant participants is by means of word clouds (Fig. 21). The example shows word cloud response profiles for the color combination that evoked the strongest responses. The word cloud representation yields an intuitive image of the 'fringe' of the response.

A more global view is obtained when plotting the pairwise correlation matrix of response profiles (Fig. 22) for the group of concordant participants. Note the block structure, which is apparently related to chord complexity. There is evidently some systematic pattern; the various combinations appear to come in groups. The proper way to investigate this is through clustering and dendrograms. The result will depend on the similarity metric. The coefficient of variation is an obvious choice. 


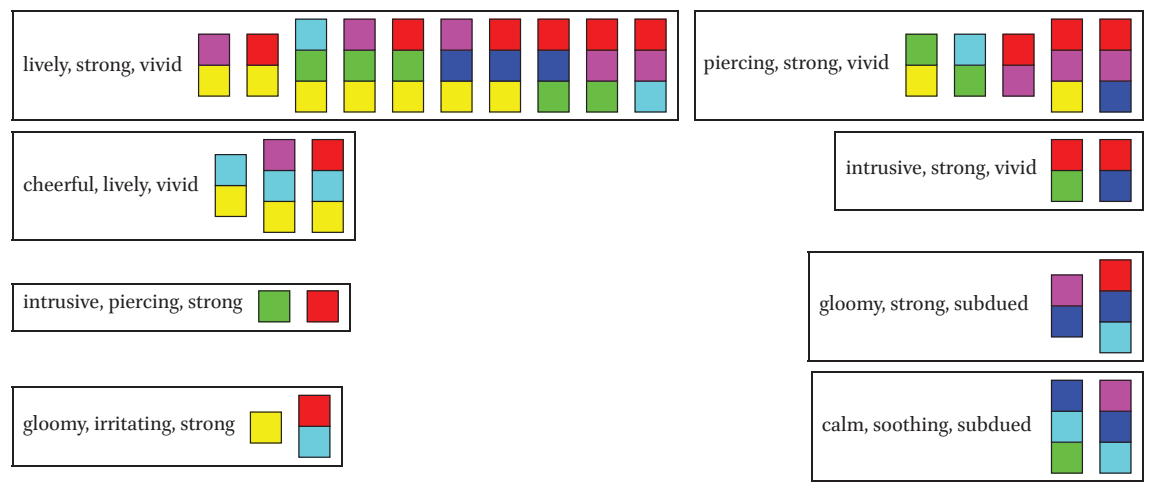

calm, gloomy, strong

Figure 20. Profile clusters for the concordant group. Only cluster with two or more members are shown. (Note that we omit the white-black in chromatic combinations. Remember that all combinations contain KW.)
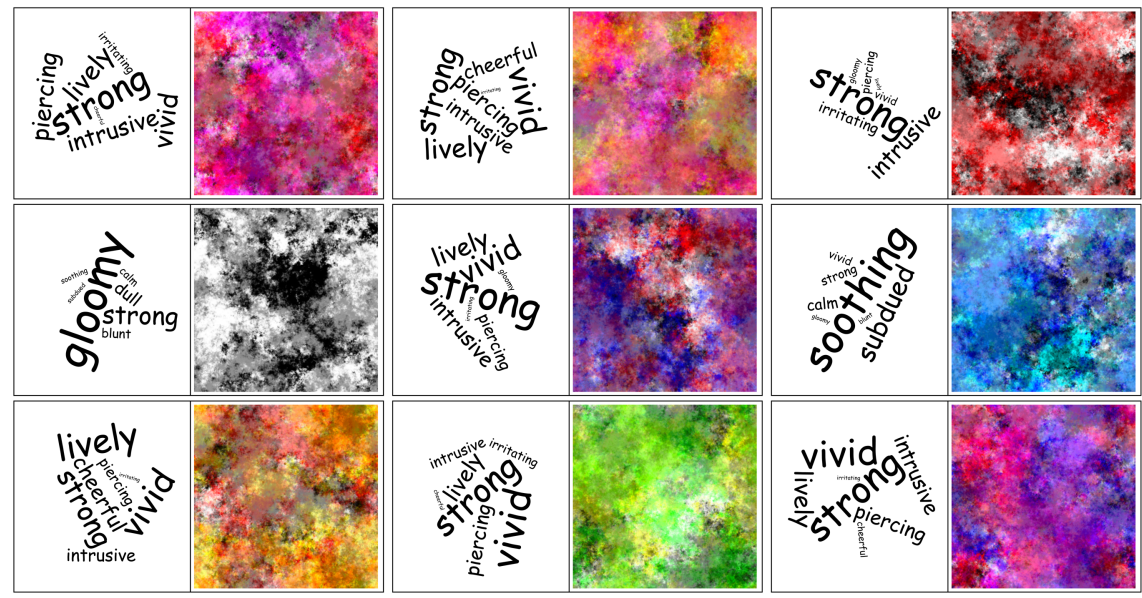

Figure 21. Word cloud profile responses for the concordant group. Here we show the profiles of the combinations that evoked the strongest responses.

Of course, one might also attempt to extract structure through principal components analysis(PCA) or independent components analysis (ICA) (Hanada, 2013). We abstain from factor analysis because we do not want to impose conventional color harmony notions on the data. The main point of interest concerns the singular values list obtained in the course of PCA. This 


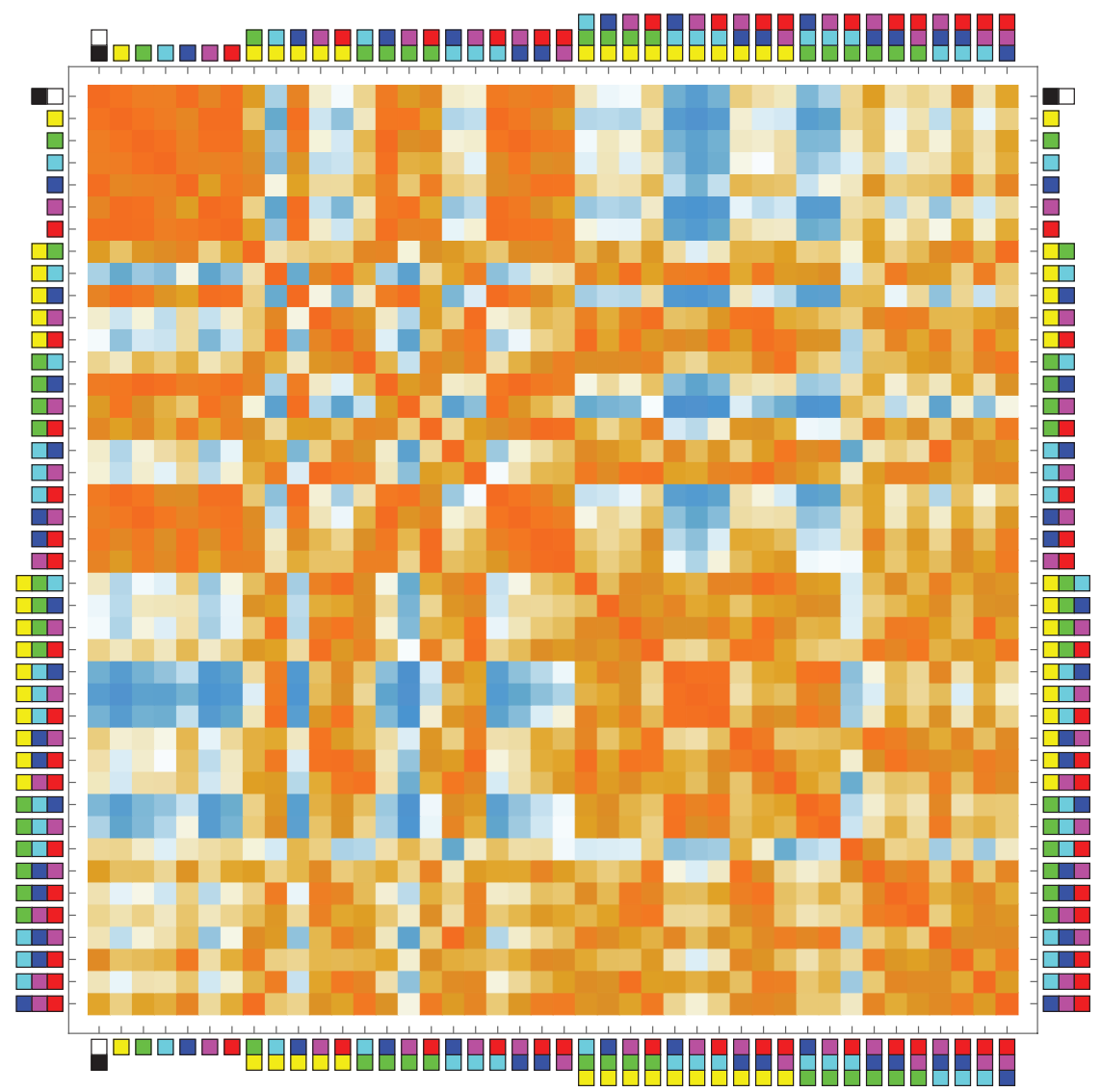

Figure 22. Pairwise correlation matrix of response profiles for all color combinations. This is for the concordant group. More orange indicates more positive (the diagonal has the maximum value), whereas more teal indicates more negative values. Insignificant correlations are near white. There is much 'noise;' two singular values explain $90 \%$ of the variance.

indicates how many distinct patterns might reasonably be extracted from the data.

\begin{tabular}{lc}
\hline degrees of freedom & variance explained \\
\hline 1 & 0.539367 \\
2 & 0.726809 \\
3 & 0.861485 \\
4 & 0.926218 \\
5 & 0.958913 \\
6 & 0.983969 \\
7 & 1 \\
\hline
\end{tabular}



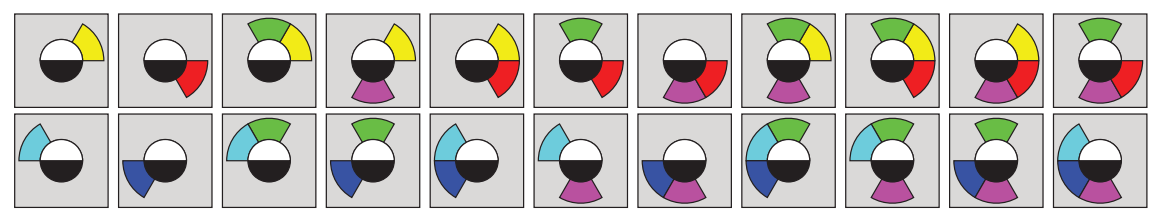

Figure 23. Result of clustering using the $K$-Means algorithm with Euclidean distance and standard deviation criterion function, asking for two clusters. Notice that the two clusters (top row and bottom row) split perfectly according to the traditional cool-warm criterion. The clustering was on the responses of the concordant group for all color combination that were either cool or warm.

Given the variability we estimate that it might be reasonable to extract three or four patterns. The PCA or ICA patterns themselves are not very useful, because they are bipolar by construction.

A straight clustering of the data for the concordant group into two clusters, using $K$-Means with Euclidean distance and standard deviation criterion function yields the result shown in Fig. 23. The clustering was on the responses of the concordant group for all color combinations that were either cool or warm. We find that the split is perfectly according to the cool-warm criterion, using the augmented definition (neutral may join either side). There is not a single exception. This is a very important result. The cool-warm dichotomy is so universal in art and design that a different outcome would be highly problematic.

If we do the same clustering on all items for the concordant group that are neither all cool, nor all warm, we obtain a very different result. The achromatic pattern is one cluster, all others in the other cluster. Of course, this is another clustering result that clearly makes sense.

Other ways to try to make sense of the data is to look at the responses for collections of color combinations for the concordant group that might be expected to be 'special' in some way. If so, then it is something to take note of, if not, then the data give little support to the notion that the established concepts make much sense (Supplementary Section S8). Either outcome would be fine. After all, the established notions are not explicitly based on empirical evidence. If it seems so, then this might be no more than a self-fulfilling prophecy. We take a quick look at a few issues.

In Figs 24-26 we show the case of monochromatic combinations for the concordant group. Apparently, there is quite a bit of structure here, although it is hard to interpret. For instance, we see no immediate connections with the color preference data.

Another case are the analogous schemes (Figs 27, 28) for the concordant group. These are very important in the arts, indeed almost universally applied in paintings, albeit often with a complementary accent. 


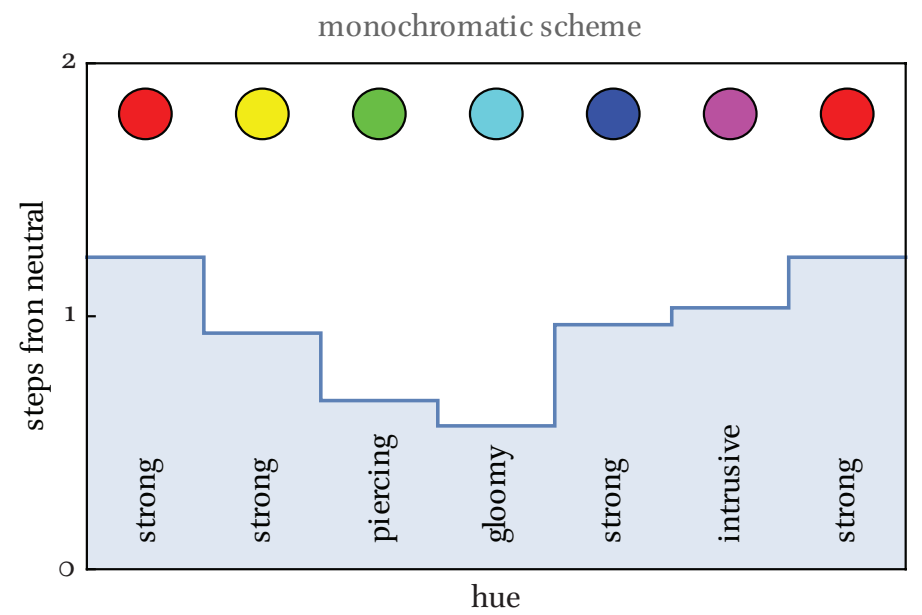

Figure 24. Monochrome responses for the dominant category as a function of the hue order in the color circle for the concordant group. Note that there appears to be a smooth transition in the magnitude of the response. Compare Fig. 25. The 'gloomy' response to cyan is still seen in the word cloud for blue, but is (almost) absent in the word cloud for green.

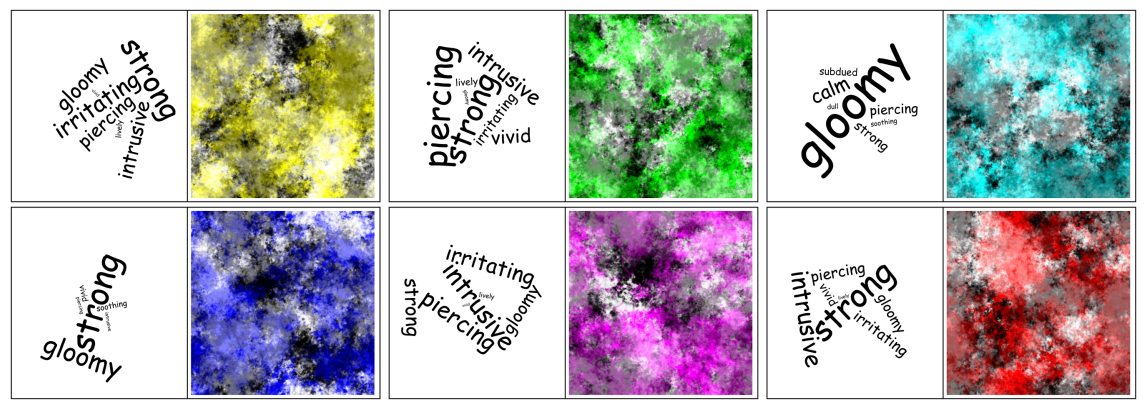

Figure 25. Monochrome responses as a function of hue for the concordant group. Compare Fig. 24, which gives the strengths of the responses, and Fig. 26, which shows pairwise clustering tendencies.

In Fig. 28 we show the relevant data for the concordant group. In several cases the responses are quite different. However, one might feel that 'vivid' and 'strong,' or 'piercing' are not that far apart.

Other instances are the complementary and triadic schemes (Fig. 29) for the concordant group. Complementary schemes are either 'gloomy' (the coolwarm pairs), or 'irritating' (the neutral pair). These are strong affections, which might well account for the popularity of such schemes.

Perhaps surprisingly, the triadic schemes are 'lively' (RGB) or 'cheerful' (CMY). Although complicated schemes they give rise to positive affections. 


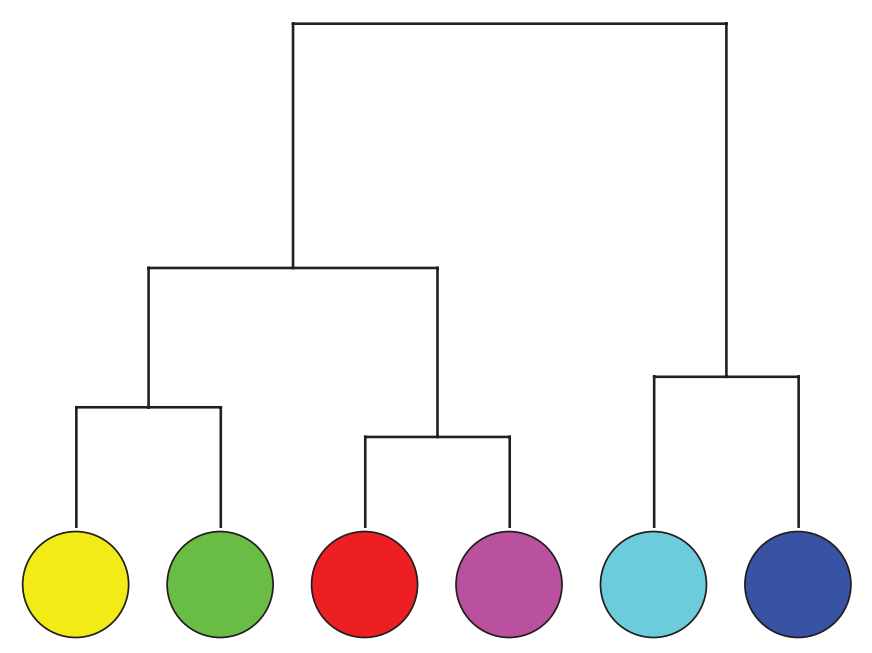

Figure 26. Dendrogram for the monochromatic profiles for the concordant group. Cool and warm are nicely separated at the first node. The other nodes are on the warm side of the tree. Green is seen to split off from yellow, purple from red (compare also Fig. 25). The resulting pairs (lowermost levels) are very common choices for analogous schemes (see also Fig. 27).
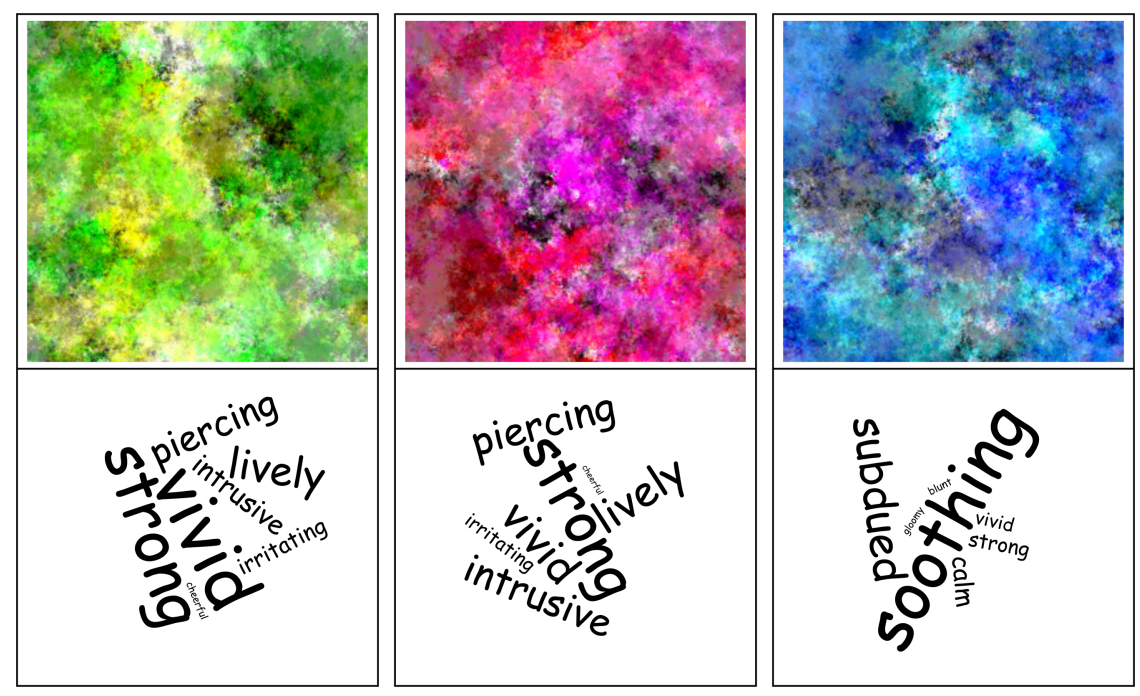

Figure 27. Some dichromatic patterns suggested by the dendrogram of monochromatic schemes (Fig. 26) for the concordant group. 

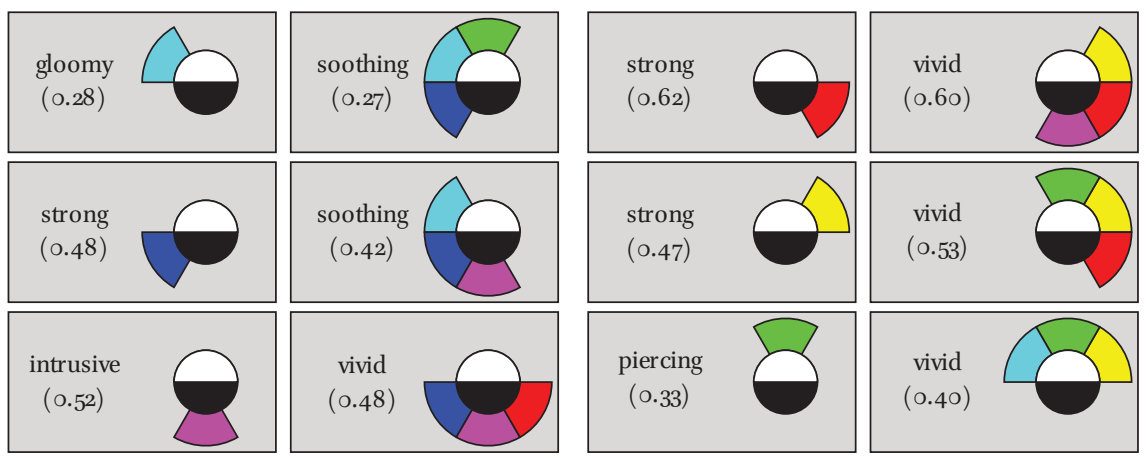

Figure 28. Left analogous cool, right analogous warm combinations for the concordant group. Widening the range makes very little difference. [The numbers are the average responses on the $(-2,+2)$ Likert scale.]
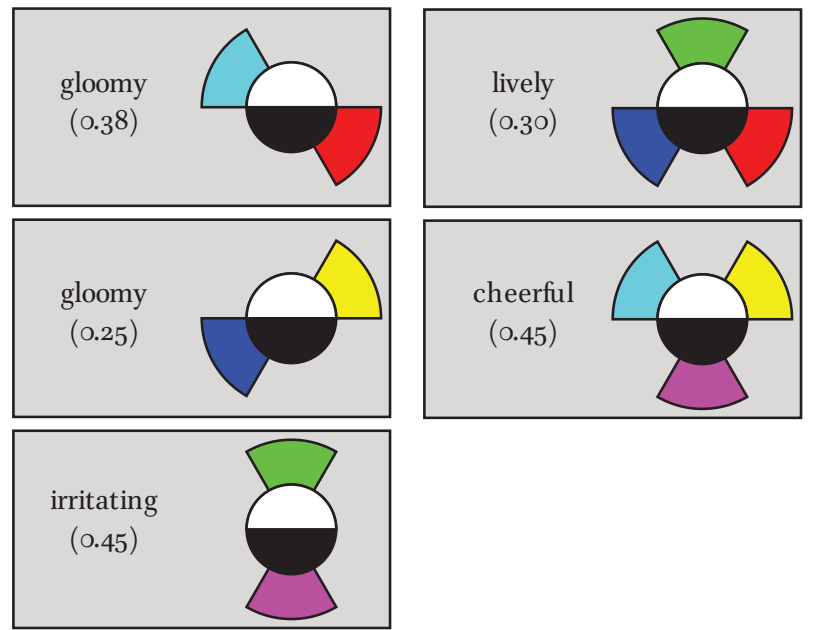

Figure 29. The complementary (left) and triadic (right) schemes for the concordant group. Why the jump from 'gloomy,' 'irritating,' to 'lively,' 'cheerful?' Of course, our data cannot provide an answer to that. [The numbers are the average responses on the $(-2,+2)$ Likert scale.]

Especially a comparison with the complementary schemes makes one wonder from where the difference derives. Of course, in practice these schemes are bound to interact strongly with other pictorial cues.

\subsection{Findings for the Full Group of Participants}

The findings for the full group are very similar to these of the concordant group. 
For instance, the strongest responses (Fig. 21) are essentially the same, so are the monochrome word clouds (Fig. 25). The cool-warm clusters (Fig. 23) are identical and the monochromatic dendrogram (Fig. 26) is the same.

The correlation matrix (Fig. 22) is very similar, but more 'noisy,' which is especially noticeable for the complicated combinations (the bottom right corner of the figure). The profile clusters (Fig. 20) are different, although many similarities are noticeable.

The focus on a (large) group of concordant participants is useful, because it shows (by definition) cleaner data. The major conclusions do not critically depend upon it. Apparently the non-concordant group is heterogeneous, so it appears in overall data as 'noise.' This notion is corroborated by cluster analysis. The concordant group indeed appears as a major cluster, but there is not a second coherent cluster, just a left-over.

\section{Analysis of Mosaic Patterns}

The basic analysis is essentially the same for the mosaic and the texture patterns. We again find an evident split in the group of participants. The concordant groups for the two cases overlap heavily (Jaccard index 0.58; Supplementary Section S6), but they are not the same. For the sake of comparability we use the concordant group identified for the texture patterns also for the mosaic patterns.

Since obvious differences are not apparent, one might simply ask how well the texture data predict the mosaic data. We find that the range of the correlation per participant over all combinations and categories is $[-0.04,+0.51]$, quartiles $(0.17,0.25,0.30)$. Not great, but there is an obvious relation.

Are there systematic differences with respect to pattern or category? When averaged over participants and combinations, we find that the correlation of the distributions over categories amounts to 0.92 . When averaged over

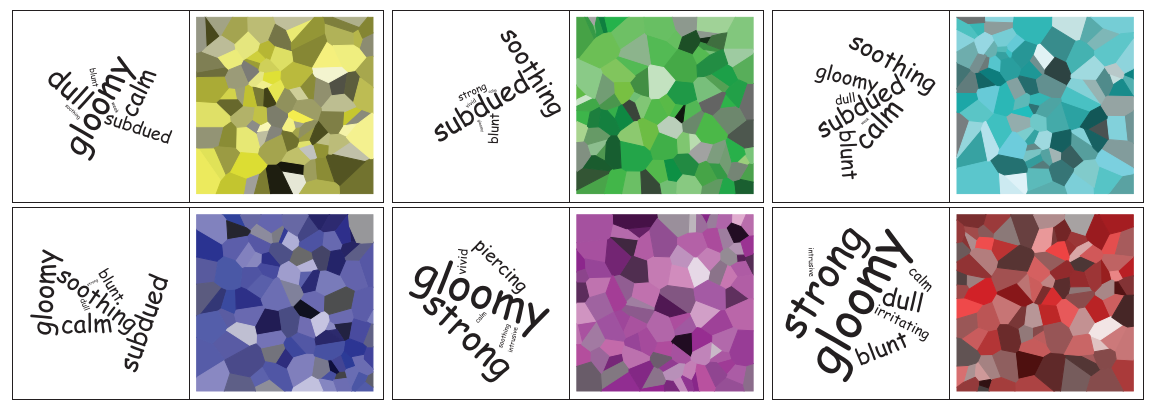

Figure 30. Word clouds for the monochromatic mosaics. (Compare Fig. 25. Note that only the relative size is important in the word clouds, don't take letter size as absolute.) 
participants and categories, we find that the correlation of the distributions over the combinations is 0.51 .

The pattern of differences appears diffuse, we failed to identify any systematic trends. As an example we show the word clouds for the monochromatic mosaics (Fig. 30, compare Fig. 25).

\section{Foreground and Background}

This particular experiment was added late in the investigation. It was run on a different set of observers. The composition of the group was similar to that of the main group. We did not detect differences in statistical structure. This follow-up experiment has no further relation to the main one, it merely checks on a possible importance of foreground versus background. The topic is of immediate importance to the visual arts.

In Fig. 31 we show a dendrogram of the data. Note that the grouping is clearly by color combination. This perhaps suggests that the foreground/background distinction might only be a minor factor.

In order to investigate this in some detail we prepare bar charts of the comparisons of pairs (Fig. 32 as an example). From such comparisons it is evident that red-cyan is not the same as cyan-red. We find this to be the case for all

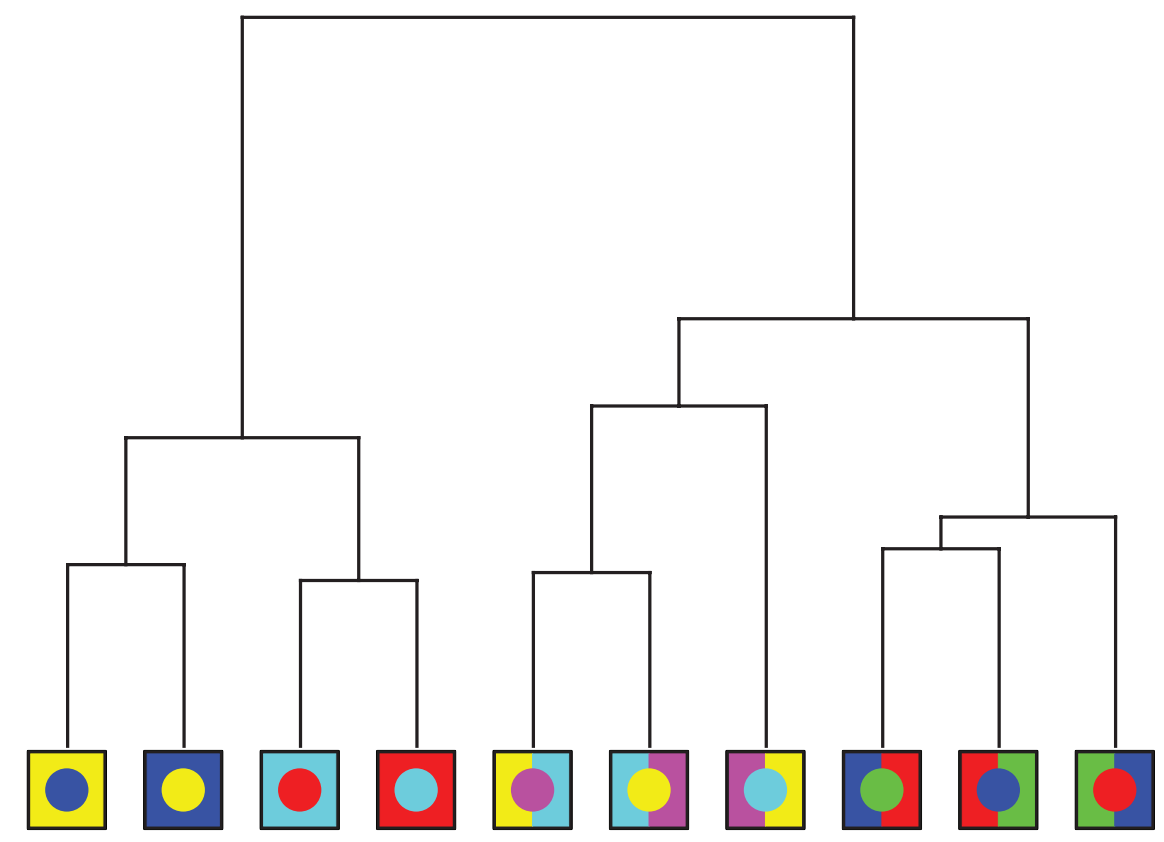

Figure 31. Dendrogram for the foreground/background stimuli. The clustering is evidently by color tuples, irrespective the foreground/background relation. 


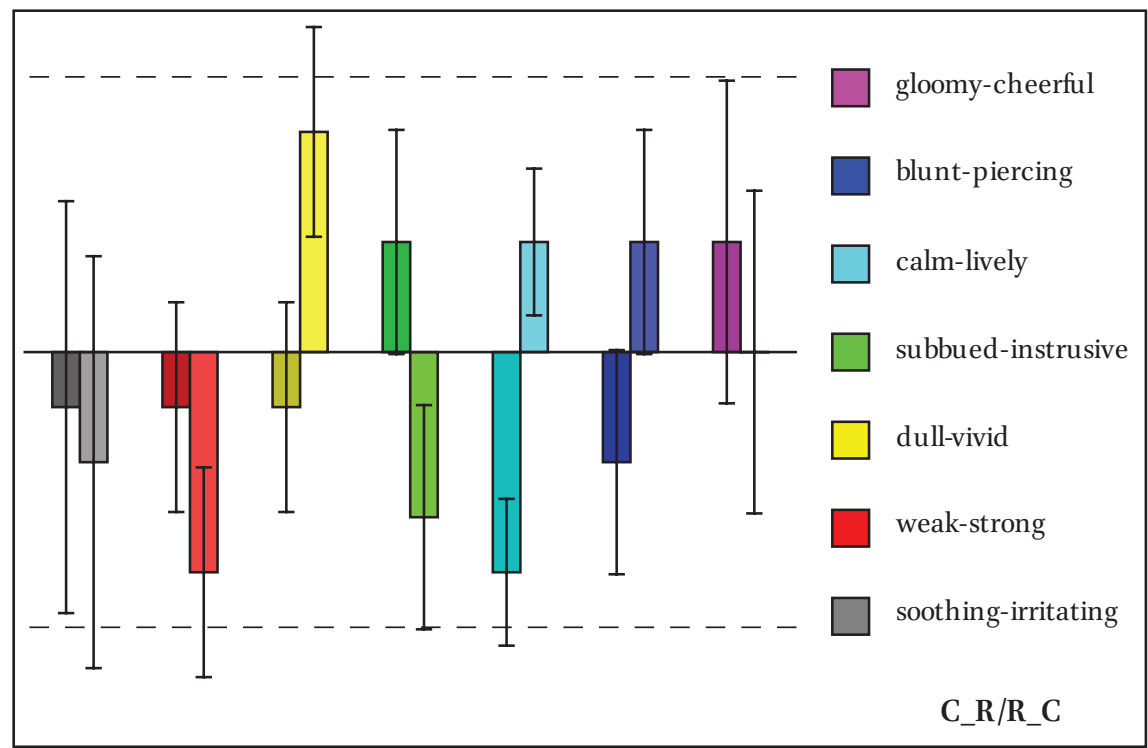

Figure 32. Bar chart for the comparison of red-cyan and cyan-red. The dashed levels indicate half a Likert-scale step. Although the pairs are the same, the foreground/background relation makes a difference. ('C-- $\mathrm{R}$ ' denotes ' $\mathrm{R}$ foreground, $\mathrm{C}$ background' and so forth.)

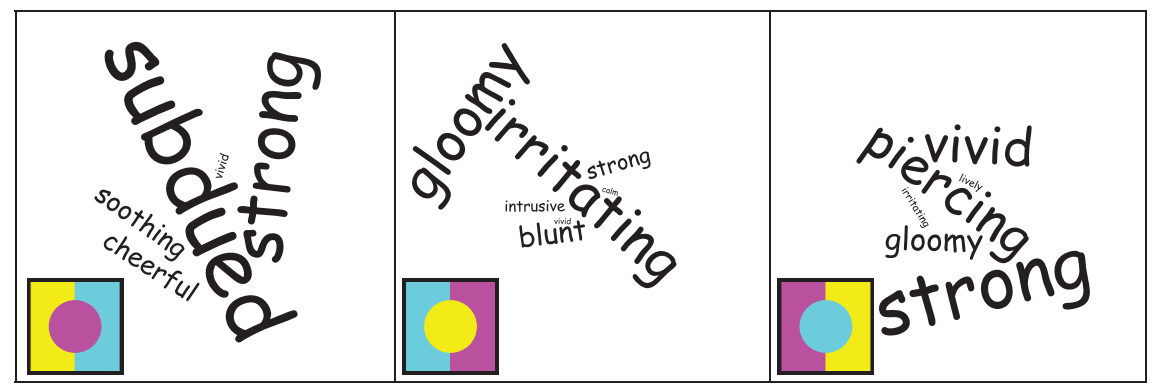

Figure 33. The distinct patterns for the triple MYC.

instance pairs. As an example we show the case of MYC, with representations YC-M, MC-Y and MY-C (Fig. 33).

Apparently the foreground/background dichotomy makes a difference to the affective response; it is not merely the chromatic structure that counts.

\section{Speculative Analysis Inspired by Artistic Lore}

Many apparent 'confusions' we tend to detect in clusterings or dendrograms involve red-magenta, blue-cyan and green-yellow pairs. We speak of 

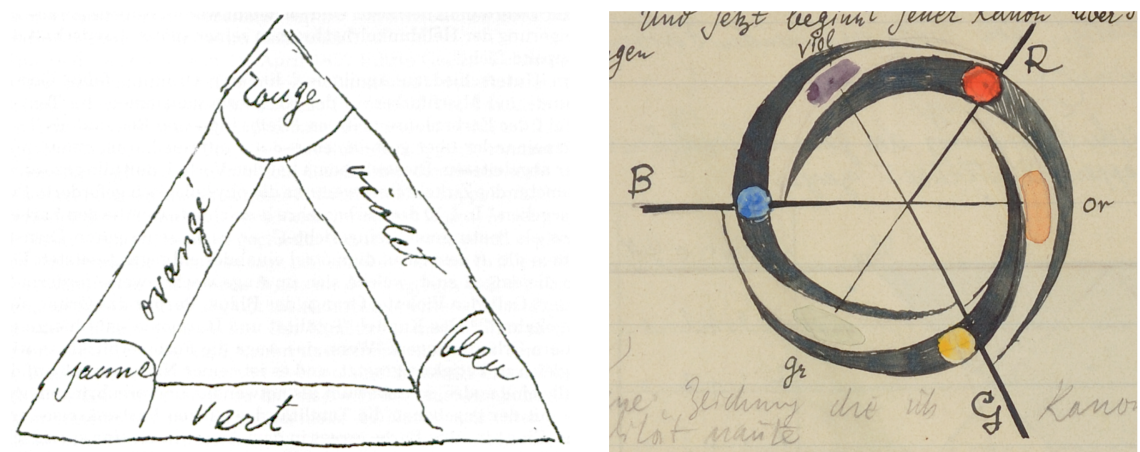

Figure 34. At left, Delacroix (1834) (from a notebook). At right, Paul Klee, Kanon der farbigen Totalität (1931; drawing from Bildnerische Gestaltungslehre, Zentrum Paul Klee, Bern, Switzerland). The orange and violet patches are noticeable, but the green patch is quite vague - possibly the paint bleached over the decades. Notice the difference between 'G' (yellow, from Gelb) and 'gr' (green, from Grün).

'confusions' when correlations on both members of random bipartitions of the data tend to disagree.

Moreover, for the monochromatic patterns we notice clustering of red with purple, blue with cyan and green with yellow (Fig. 26).

This brings to mind the fact that from the nineteenth century on the "primary colors' for the artist have been red, blue and yellow. Figure 34 shows two random examples, numerous others could be quoted. These date from 1834 (Delacroix) and 1931 (Klee), nearly a century apart.

Delacroix's color triangle has vertices Red (rouge), Blue (bleu) and Yellow (jaune), with Green (vert) on the Yellow-Blue edge, Orange (orange) on the Yellow-Red edge and Purple (violet) on the Red-Blue edge.

Paul Klee has a color circle with Red, Blue and Yellow centers at $120^{\circ}$ intervals. These primary colors are suggested to have an influence over at least half the color circle, reminding one of Ostwald's ideas. Note 'Gr' (for green, Grün) between 'G' (for yellow, Gelb) and 'B' (for blue, Blau), 'Or' (for orange, Orange) between ' $\mathrm{G}$ ' and 'R' (for red, Roth) and 'Viol' (for purple, Violet) between ' $R$ ' and 'B' (Note 23).

The palette BYR became a defining factor of the Bauhaus (1919-1933) style in the early twentieth century (Note 24). It was in the air, for instance, the Dutch 'De Stijl' movement was founded in Leiden in 1917 (Fig. 35; Overy, 1991; Note 25).

Hence the intuition:

What happens when we reckon any color as subordinate to BYR?

Thus, what when we reckon purple as red, green as yellow and cyan as blue? At first blush this is probably a strange idea. 


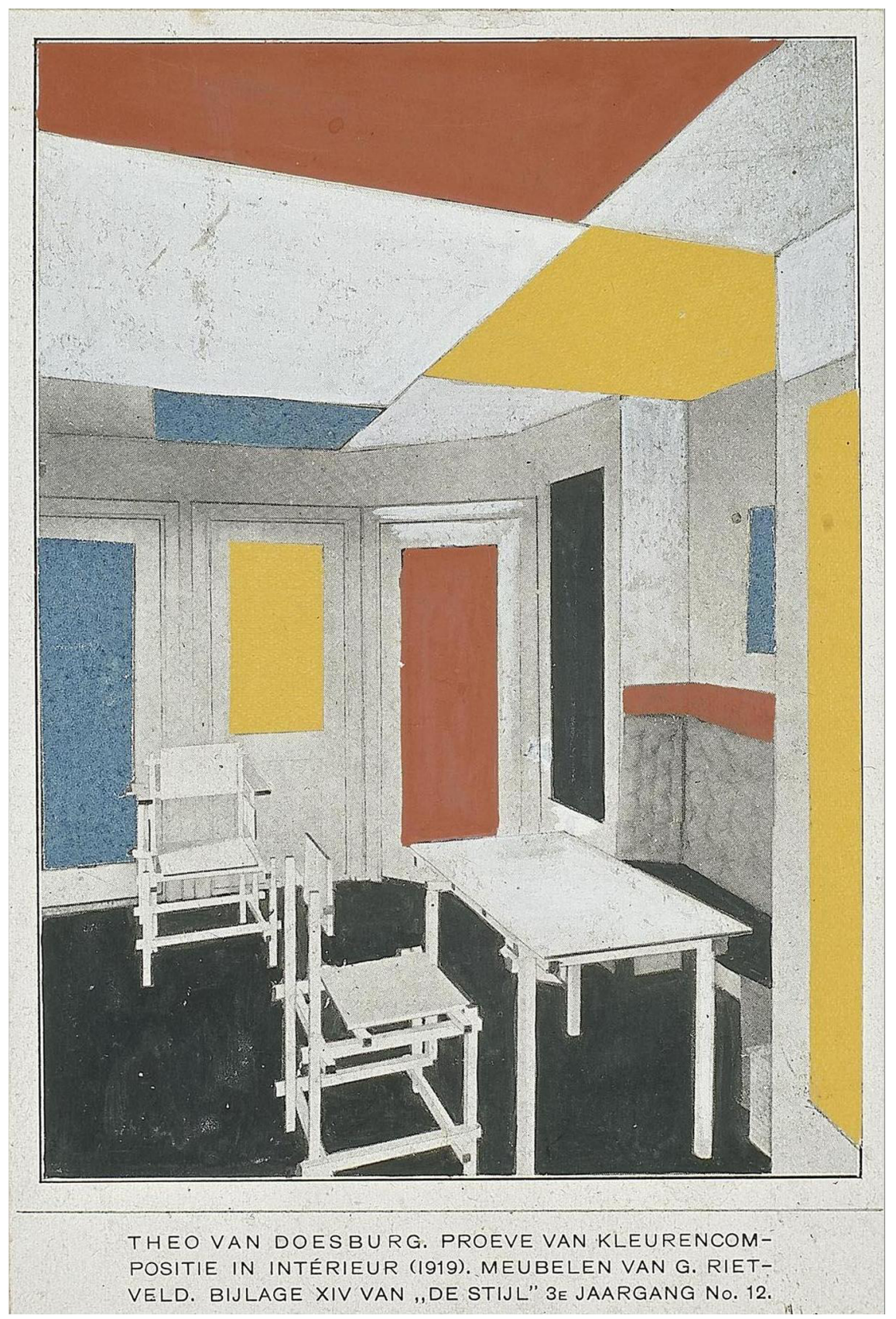

Figure 35. Van Doesburg and Rietveld interior (1919). Black, gray, white, blue, yellow and red. 


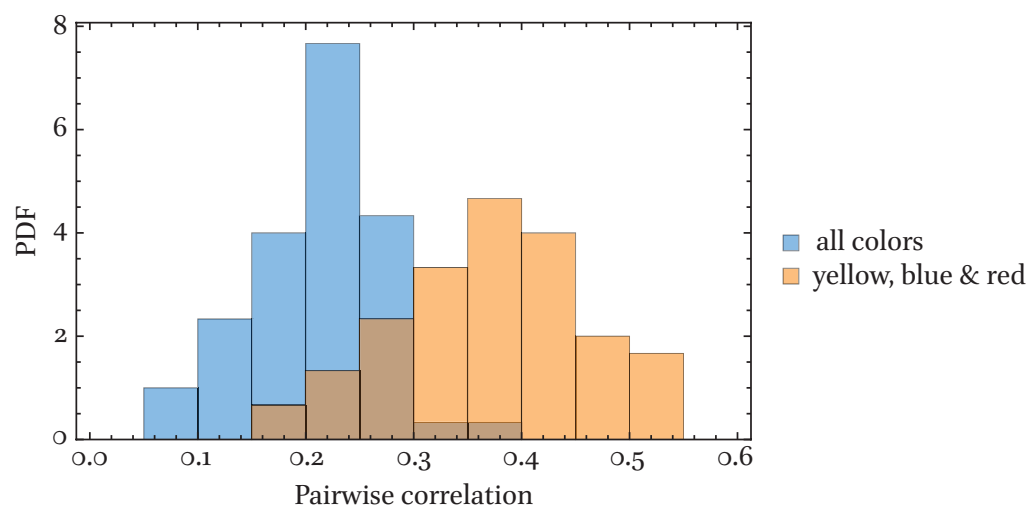

Figure 36. Histograms of correlations of responses of pairs of participants over all combinations. This involves all 60 participants.

If we do so anyway, the number of distinct combinations in our stimulus set drops from 42 to 8 . We can easily convert the data to this simplified representation by selective averaging. (For instance, the combinations KWCM, KWCR, KWBM, KWBR, KWCBM, KWCBR, KWCMR, and KWBMR all collapse to KWBR.)

As a result, the participants become mutually much more similar. There is no indication for a bipartition of participant response patterns. Thus, it is no longer necessary to split off a concordant group. Median of correlations between pairs of participants is 0.39 (interquartile range 0.33-0.44), much better than the 0.21 (interquartile range 0.18-0.25) for the complete data (Fig. 36). A dendrogram for the BYR-projected complete data is unsurprisingly very much simplified. More importantly, it makes more sense from the artist's perspective (Fig. 37).

The overall simplified correlation matrix is very similar to the detailed one (Fig. 38). Again, two singular values are sufficient to explain over ninety percent $(93 \%)$ of the data. Is this finding at all remarkable (Note 26)? In order to check this quantitatively, we simulated the effect of random coarse-graining from the six cardinal colors to a mere three (Note 27). We find that the median pair correlation drops below the $25 \%$ quartile of that for the scheme based upon BYR priority.

Only a few instances are on par, but these are trivial modifications, for instance setting red to magenta instead of magenta to red and so forth. Such are equivalent cases, one cannot distinguish between them. Instances that have a relatively high median pair correlation (although not on par with the optimum) are all partly related to the optimum (Fig. 39). They have one or more 


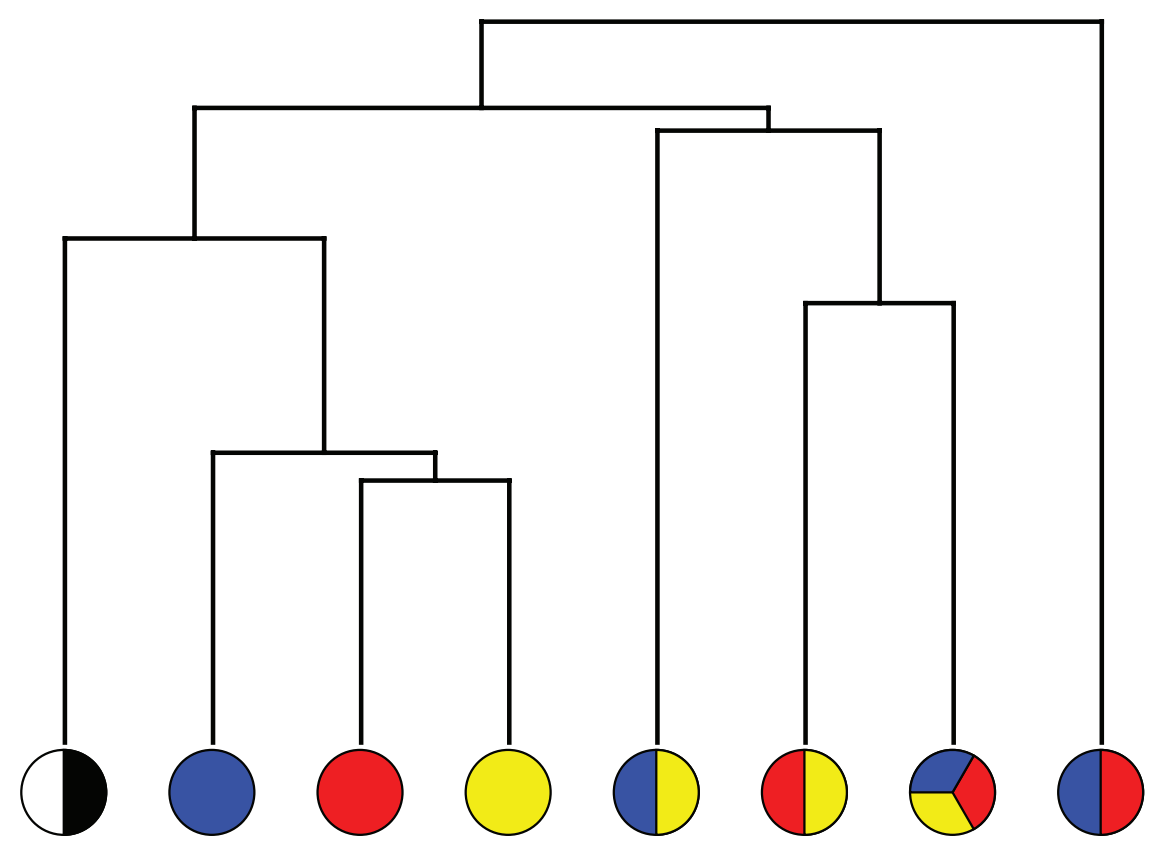

Figure 37. The dendrogram of all data summarized by equating Red with Magenta, Green with Yellow and Cyan with Blue.

changes that break the topology of the color circle, such as a red-cyan interchange.

Thus, the case is a clear-cut one. The projection on BYR is indeed remarkable. Apparently the artist's choice of primaries BYR - different from the colorimetrically indicated RGB - has its merits.

This analysis nicely reveals structure in our data (Figs 40 and 41). Perhaps surprisingly, it does so by placing the combinations into bins that have essentially no relation to the classical chords. This opens up a novel perspective on the issue, one that could hardly have been anticipated a priori.

Of course, in cognitive awareness green is not yellow, nor is purple red, nor is cyan blue (Figs 40, 41)! Indeed, one can spot such differences at a glance, at least for uniformly tinted patches. But then, in di- or trichromatic textures you may need a second glance to notice the difference. And in many cases, in order to note the difference between certain di- and trichromatic patterns may require scrutiny (Note 28). The BYR simplification appears to dominate the level of momentary awareness, but is overridden in cognition proper.

Apparently such a coarse-graining is conducive in making sense of the data in the context of our experiment (Figs 37, 40). Much of the additional structure offered by a finer-grained approach only leads to confusion due to 


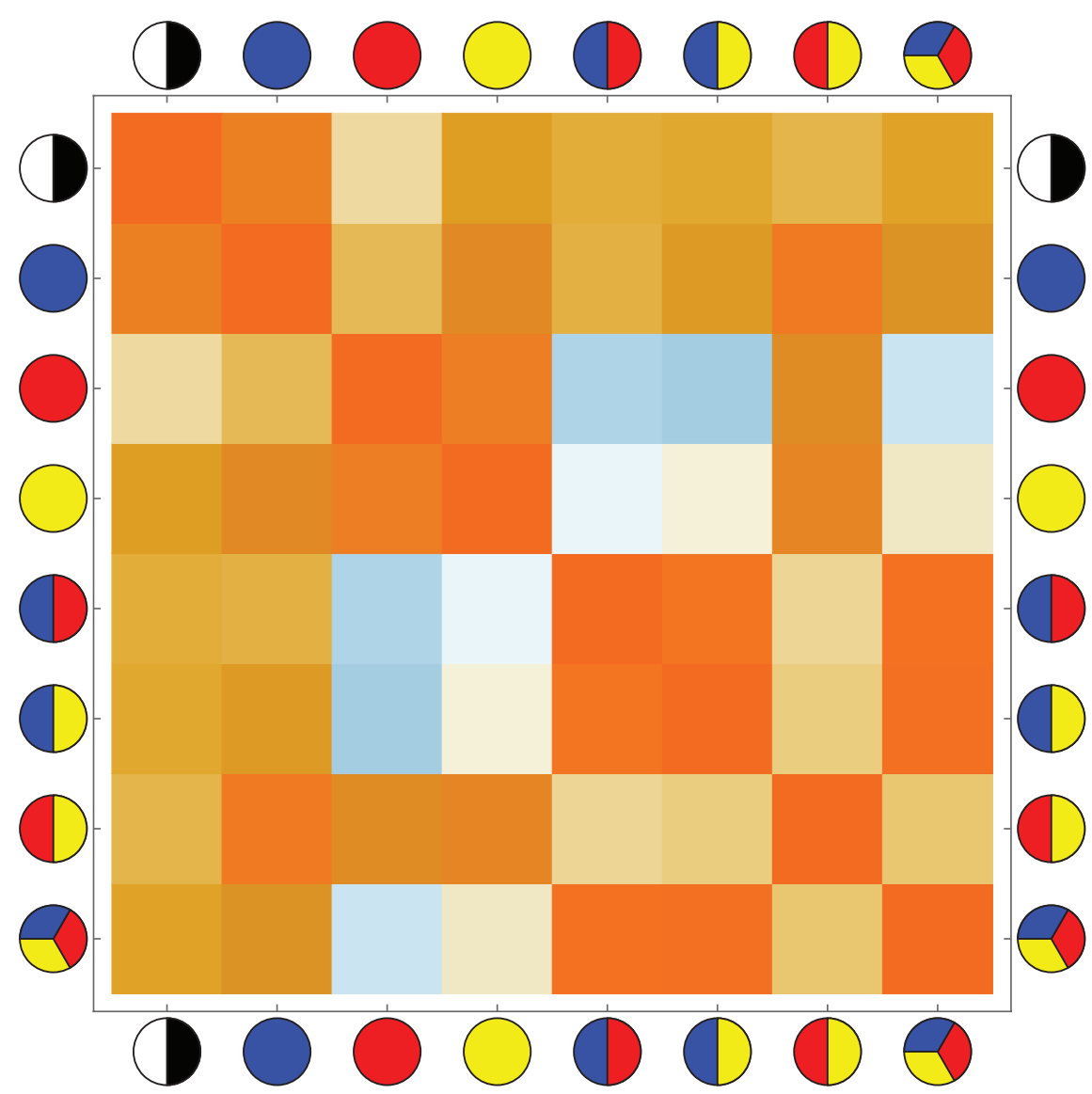

Figure 38. The simplified correlation matrix (compare Fig. 22).

idiosyncratic differences in a population of a few dozen naive participants. The coarse-graining reveals the roots.

\section{Discussion}

\subsection{Color Preferences}

Our informal enquiry probably triggered cognitive (Derefeldt et al., 2004), culturally determined thoughts (for a perhaps more appropriate method see Clarke and Costall, 2008) in the participants.

Moreover, it almost automatically focuses on colors as abstract entities, for some participants perhaps mere words, for others perhaps fuzzy colored patches in visual imagery. 


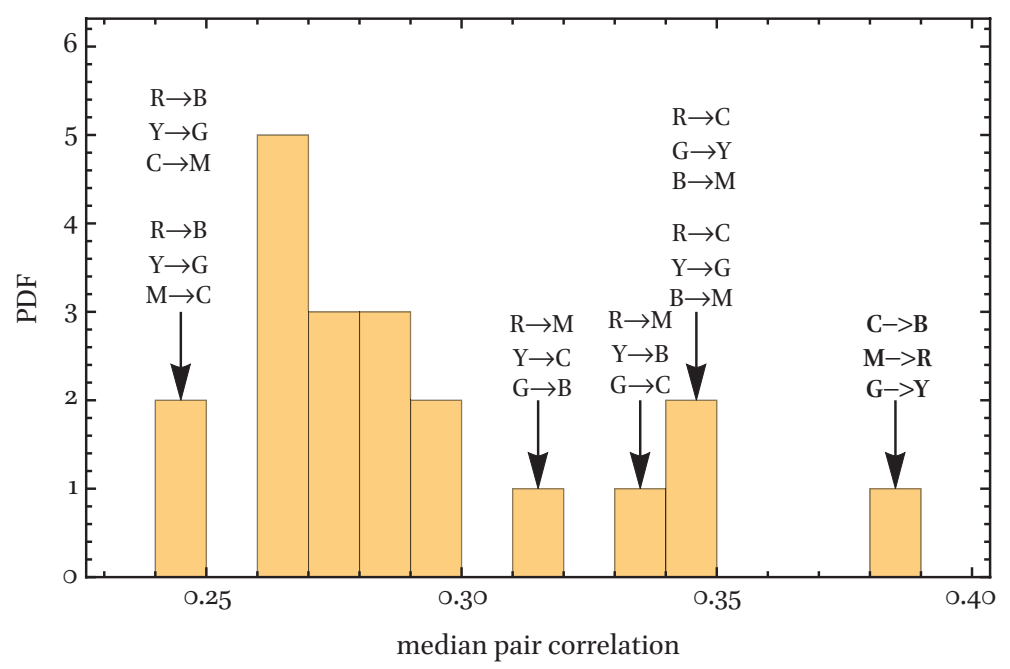

Figure 39. Median pair correlations for all coarse-graining schemes. The proposed scheme leads to the highest overall concordance, it is a clear winner. This involves all 60 participants. Lower correlations interchange (near) complementaries like red and cyan.

Color preferences in real life typically derive from situational awareness (the light-field, the chromatic envelope), or objects (Geymonat de Destefani and Whitfield, 2008; Koenderink et al., 2015; Palmer and Schloss, 2010; Schloss et al., 2013; Smith, 2008; Tangkijviwat et al., 2010). Preferences depend not only on hue (Schloss et al., 2020). However, most likely participants had fully saturated highly chromatic colors in mind.

We did have surprisingly few (Clarke and Costall, 2008) initial refusals to come up with a preference and few participants opted for an achromatic color. No signs of chromaphobia. The overall result is that people prefer blue and perhaps somewhat dislike green, purple, yellow or red.

If we apply the BYR coarse-graining, then saying that people like blue, dislike yellow and red covers most of the action, with very few exceptions. It is hard to say to what extent such preferences are culturally determined, exist on the cognitive level, or have deep roots in visceral processes (Batchelor, 2000; Son et al., 2020).

Looking into more detail, we find significant differences between gender and age groups. Apparently a full story would be complicated. There is a large literature on these issues. Our informal findings fit seamlessly into mainstream knowledge (Bakker et al., 2015; Beke et al., 2008; Note 29).

The reason we asked for preferences was that these might conceivably have consequences for the actual experiment. In retrospect, we find no indications for that. Thus, the preference data played no further role in our analysis of the experimental results. 


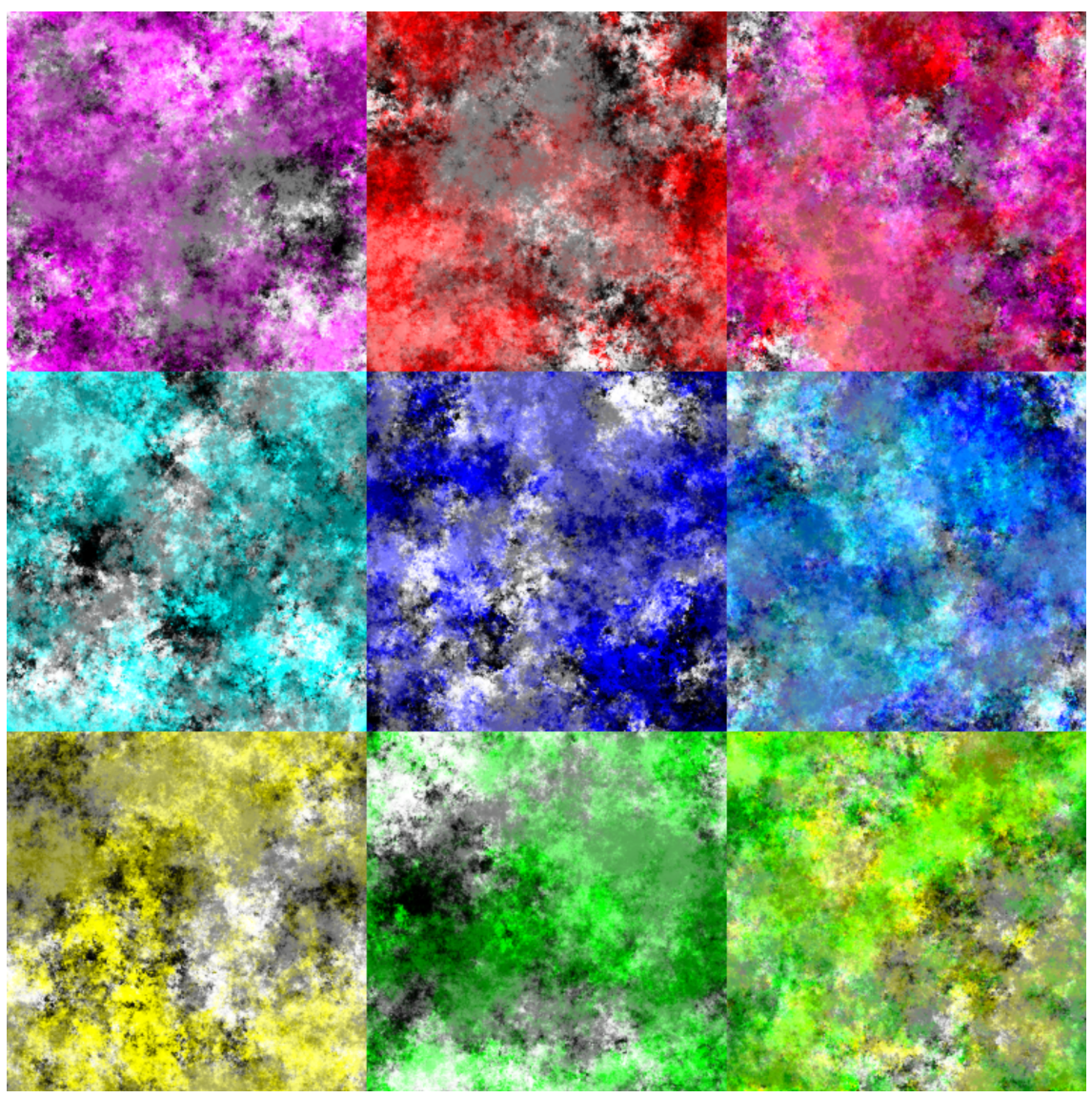

Figure 40. Red (top row), blue (center row) and yellow (bottom row). Coarse-graining is something to get used to. However, the row-wise family resemblance is clear enough. All these patterns are rated 'strong,' but with different annotation: blues are 'gloomy,' reds are 'intrusive' and yellows are 'intrusive' to 'irritating.'

We also started this way in order to move the participants to the right mind frame ('next hour is about color'). Of course, we cannot check whether it worked.

\subsection{Affective Responses}

We use seven five-point Likert scales of bipolar categories: 'soothing-irritating,' 'weak-strong,' 'dull-vivid,' 'subdued-intrusive,' 'calm-lively,' 'bluntpiercing' and 'gloomy-cheerful.' These are aimed at 'affective judgments.' We try to steer clear of æsthetic, as well as psychophysical judgments.

We think of 'judgments' as behavioral responses based on gut feelings. We are not so much interested in linguistic, conceptual, scientific or logical 

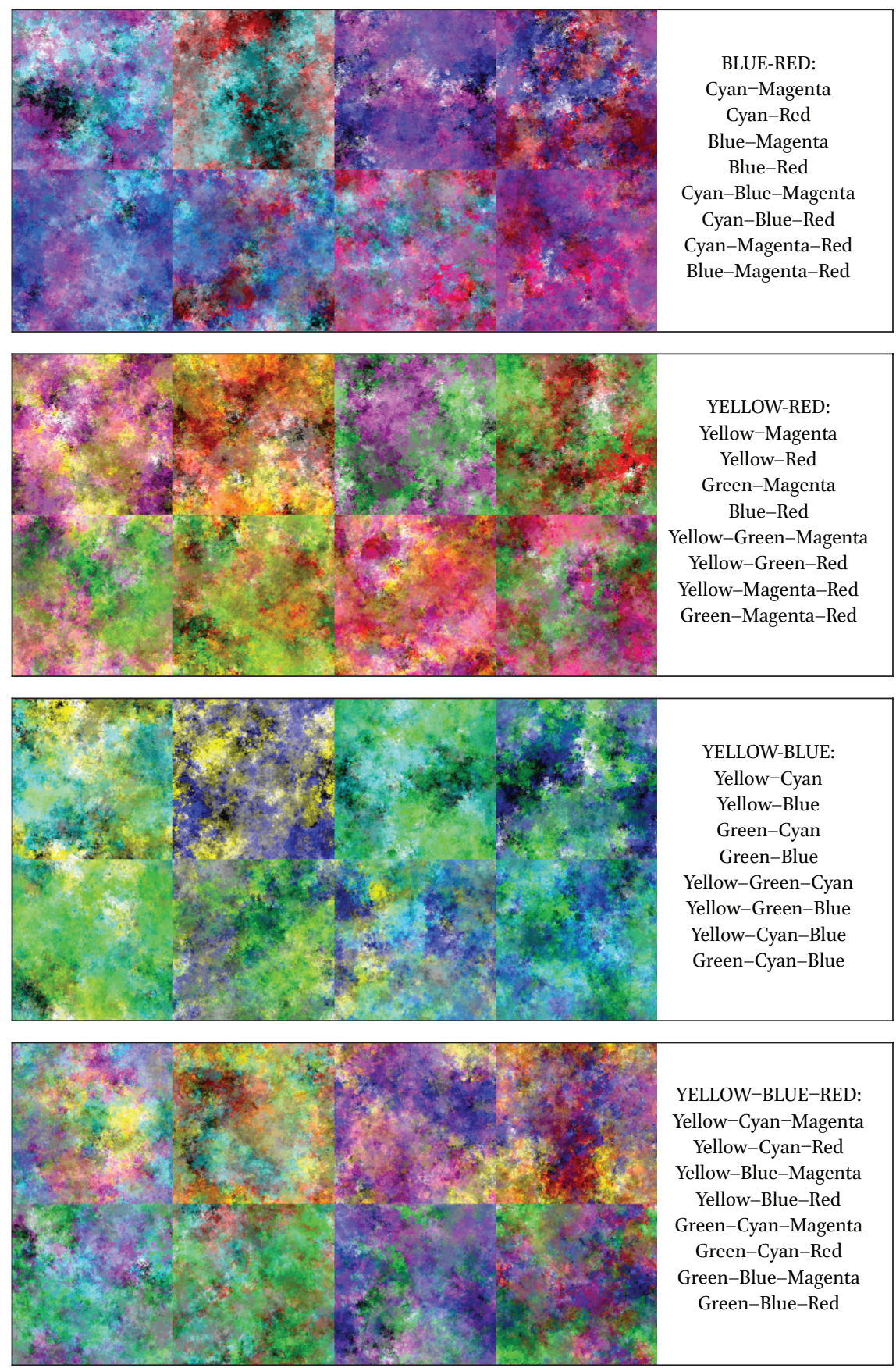

Figure 41. The remaining combination families restricted to BRY. There are 32 instances here, more than three-quarters of all combinations. From top to bottom these are predominantly 'intrusive, strong, vivid,' 'lively, strong, vivid,' 'piercing, strong, vivid' and 'lively, strong, vivid.' 
judgments. Thus, we attempt to tap sentience (feelings, intuitions, imagery, ...), not sapience (cognition proper). This may not be fully achievable due to cultural, semantic factors. For instance, in our environment funerals are often associated with the color black, museum walls with the color white. However, one may try.

Affective feelings belong to the sentient level of mind (Koenderink, 2019). Thus, they have nothing to do with the conceptual, logical, linguistic level. That is why love and hate cannot exist in isolation, that strong feelings of love and hate may alternate or that love and hate may actually co-exist in a single mind. This is not something academic that one might as well ignore, for much - perhaps most - of the overt behavior is guided by the sentient level. The 'gut level' is highly instrumental on almost all echelons of daily life. A 'perceptual judgment'(Note 30) has to result in either 'love' or 'hate' because of tertium non datur. Sentience does not recognize the Law of the Excluded Middle. Intuition operates by analogy, but anything goes. Like love-hate.

Thus, the 'Likert scale' (Likert, 1932) has very diffuse ontological roots. We use polar terms similar to hate-love, but what if opposites may meet? Does it make sense to compare, average, or correlate (all the same problem) scale levels at all? Is there a 'neutral' response in the middle? Does it feel anything special to feel 'neutral?' Sometimes yes, for there is a 'love-hate' that is all but neutral (Note 31). This only makes sense in experimental phenomenology, not in behaviorism. Behaviorism is meaningless by design (Skinner, 1938), which is perhaps its strongest point as it renders it 'science.'

These are very common considerations. It is rare - maybe impossible - for them to be dealt with satisfactorily. We do not even pretend to be able to do that. Instead of that, we proceed by the simplest of means, with an absolute minimum of prior assumptions or expectations. We are fully aware that it would be impossible (or dishonest) to defend all methods formally (Note 32). That is already rendered impossible by the very nature of the data. We simply proceed and attempt to make sense of the results.

The first step in sense making on the basis of observed structure is statistics. The major strength of statistics is that it allows one to identify things that are probably not the same.

The next step, that is 'sense making,' implies making up stories that weave such probable differences into relational patterns that appeal to the discursive mind.

Such exploration is the creative part of scientific research. It is itself not science, but a prerequisite for science. Nevertheless, it is common enough to speak of 'theories,' or even 'explanations' (Note 33). That is fine (although we would prefer to speak of 'interpretation'), as long as one is aware of the ontology. The stories that go with the patterns are what make them meaningful to the inquiring mind. The stories cannot replace the facts, but the facts are 
meaningless without the stories (Supplementary Section S9 lists the facts in meaningless fashion).

There is no scientific reason to believe that a few dozen randomly selected participants would have the same affective responses to color combinations. However, we find that the data we obtain are anything but random. Instead, there exists easily detectable systematic structure. We find that it is possible to identify a fairly large (half the total) group of participants that are mutually highly concordant. The members are similar in ways that do not follow from current scientific understanding. It is just an empirical fact.

One structure that almost jumps out of the data is the warm-cool dichotomy. It evidently explains much of the overall structure in the data. This is a striking fact, because we never asked the participants about their feelings about (relative) color 'temperatures.' It is encouraging because the warm-cool dichotomy plays a major role in the visual arts.

\subsection{The 'Limited Palette' of Visual Awareness}

We already discussed Delacroix's and Paul Klee's notions of the structure of the color world in Fig. 34. These are in most respects generic for the arts, although there are - of course - variations.

Figure 42 (right) is from Charles Blanc's Grammaire des arts du dessin (Blanc, 1891), a text that was widely read by artists in the second half of the nineteenth century. The primary triangle is blue-yellow-red, a secondary orange-green- purple. Together they form a six-step color circle, with interpolations yielding a twelve-step scale.

Since the red is shown as complementary to green, it must verge on magenta. 'Blue' is complementary to orange, so it is actually a teal. The violet is likely a purplish blue.
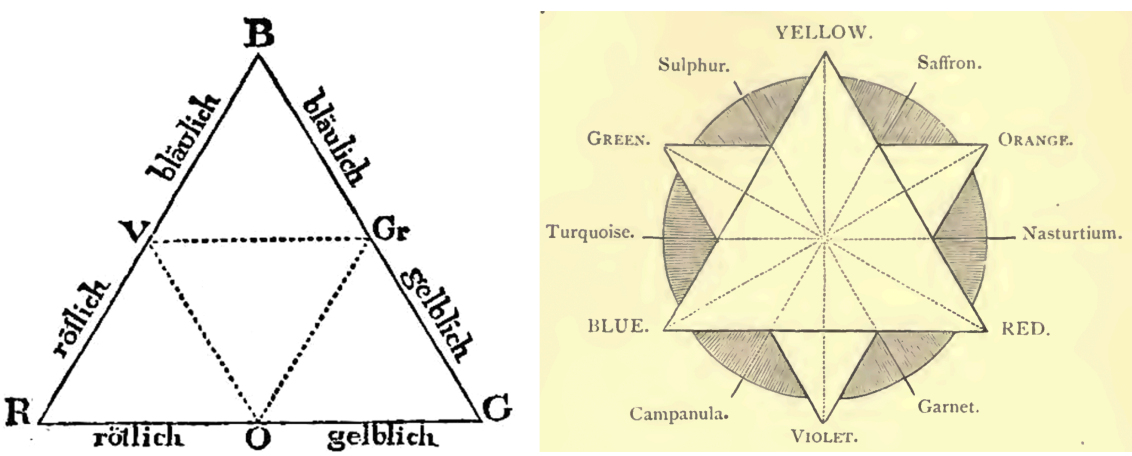

Figure 42. Left a color triangle by Philip Otto Runge (Runge, 1810); right a color scheme from Charles Blanc's Grammar of Art (Blanc, 1891). These schemes are by no means 'hollow,' like the triangle of Delacroix (Fig. 34). 


\section{UHuz meiner Darftellung crgielbt fid, folgenbes Sdyema:}

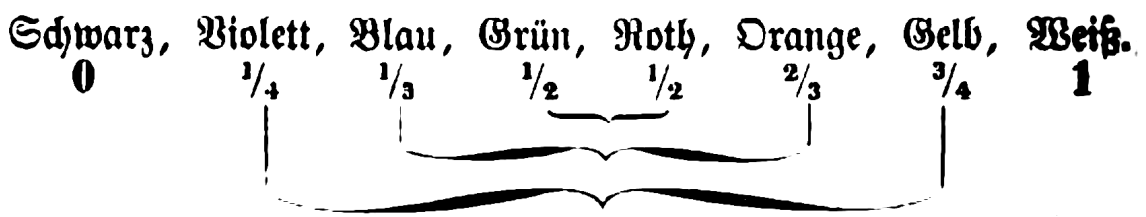

Figure 43. Bisections of the daylight spectrum according to Schopenhauer (Schopenhauer, 1816). (The top line has: "the following scheme derives from my representation:".) Such bisections are necessarily complementary. Thus, the blue (Blau) must be teal. The red must be not too different from magenta. The violet (here spectral!) must be close to blue. This maps directly on Fig. 42.

Different from Delacroix's triangle, this hue circle is not 'hollow.' This is also true for the color triangle from Philip Otto Runge (Runge, 1810; Fig. 42 left; Note 34). Here the center is 'achromatic' in the sense that Runge intends a full black-to-white scale erected at the center, out of the plane of the triangle.

Runge's triangle dates from 1810, but it is essentially the same as Blanc's (Blanc, 1891). Throughout the nineteenth century ideas changed little, Maxwell's and Helmholtz's scientific progress making no difference to artistic theory.

The scheme by Schopenhauer (Schopenhauer, 1816); Fig. 43), dating from 1816 , can be mapped perfectly on the Runge triangle. Schopenhauer (the philosopher) wrote his essay on color in order to render Goethe's intuitions acceptable to a scientifically oriented public. Runge's (the painter) understanding of color was appreciated by Goethe (the poet), who printed a letter by the artist as appendix to his Farbenlehre.

The artist's notion indeed fits perhaps better with Goethe's (Goethe, 1810) than with Newton's (Maxwell, 1857; Newton, 1704; von Helmholtz, 1855) account. Unlike Newton, Goethe considered green not 'primary.' (The Goethe edge colors ("Kantenspektren") are shown in Fig. 44.) That artists agree with this no doubt derives from the common experience that you can 'mix green from yellow and blue.' (All Kindergarten students know that!) Here the blue is clearly cyan. We also find many remarks that 'true' red actually tends to purple ('carmine,' that is \#FF0038, containing 22\% blue). Purple is generally considered as not primary, although you cannot really mix it by stirring paints together. However, it appears in partitive mixtures of red and blue (see Fig. 45).

Thus, 'complete palettes' are easily based on wise choices of white (for opaque paints; Note 35), a cool black (Note 36), yellow, a somewhat tealish blue and carmine red paints. There are many examples of painters (we 


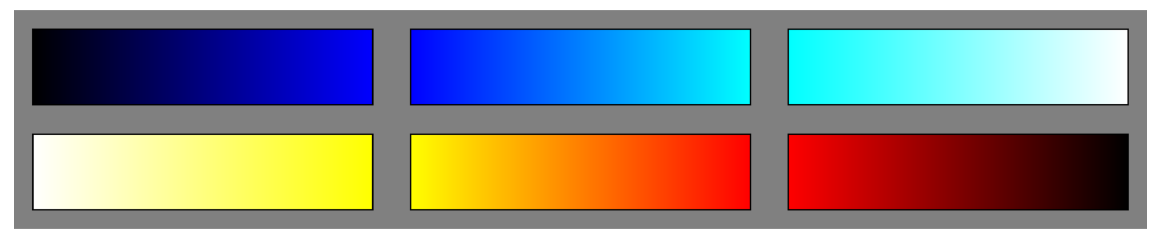

Figure 44. The Goethe edge colors (Kantenspektren). The cool edge colors are shades of blue, varieties of teal and tints of cyan, easily taken for 'blue.' The trisection shown here splits the full cool edge color series into these three subgroups. The warm edge colors are tints of yellow, varieties of orange and shades of red, easily taken for 'yellow and red.' The trisection shown here splits the full warm edge color series into these three subgroups. Looking at 'all colors' in this way suggests that if there are any primaries they should be blue, yellow and red.

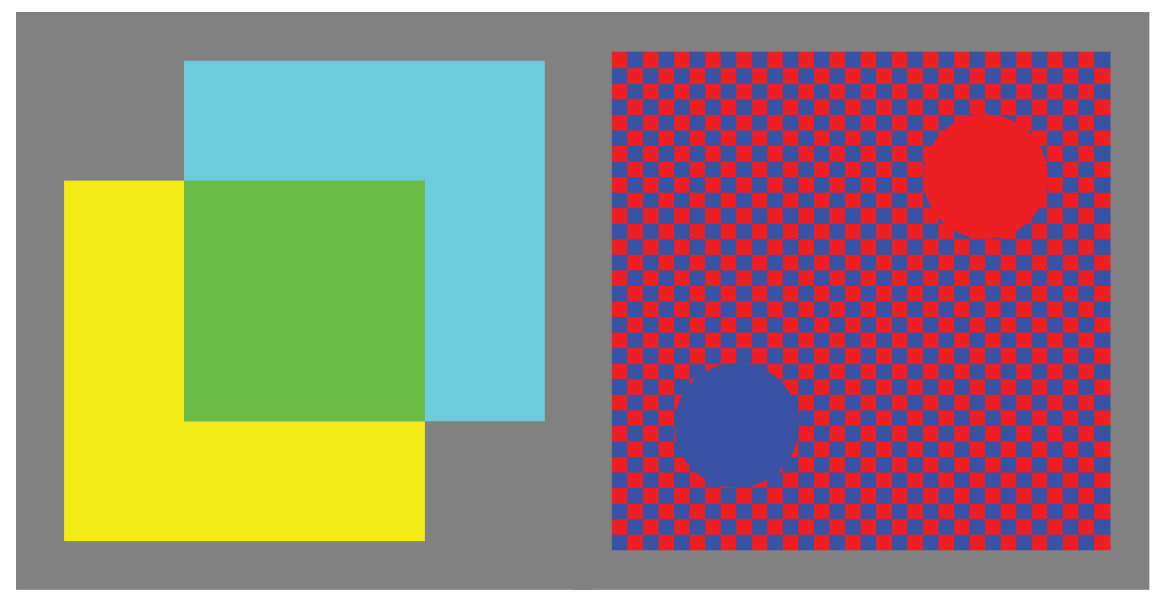

Figure 45. The green is a subtractive mixture of yellow and cyan ('blue'), the purple a partitive mixture of red and blue.

mentioned Anders Zorn) who did perfectly fine with that, although the palette would be considered 'limited' (considered positive!).

A different approach would be to have as many highly chromatic hues as possible and tone them down into tints or shades with white and black. Many painters will ignore black and obtain the same shade through mixture with a complementary (which takes experience). There are painters who work fine in this way (we mentioned Arnold Böcklin, a contemporary example is Steve Quiller; Quiller, 1989).

We consider the empirical fact that coarse-graining to blue, yellow and red tends to bypass the effects of idiosyncratic differences to be really a major finding. It was only discovered by accident during extensive data exploration. 
It could hardly have been anticipated and made scientifically respectable through the hypotheticodeductive method (Popper, 2002).

We expect this to be an important perspective in many studies into the nature of generic visual awareness. It also fits the linguistic patterns (Note 37) originally described by Berlin and Kay (Berlin and Kay, 1969; Griffin and Mylonas, 2019):

- If a language contains three terms, it contains a term for RED. (Notice that we take BLACK and WHITE for granted, all languages have these.)

- If a language has four terms, it contains a term for either GREEN or YELLOW.

- If a language contains six terms, it contains a term for BLUE.

Alternatively, Kay's Partition Rules (Kay and Maffi, 1999) have that the sequence is firstly black and white, secondly cool and warm and, thirdly red. The Böckling painting (Fig. 46) essentially has it all (BYR). Note that cool and warm is most nearly teal-and-orange, in our description blue-and-yellow. It is indeed defensible to hold that the artistic blue-and-yellow is really teal-andorange. Artistically this is the major dichotomy (Fig. 13). It is not possible to check that from our data, but it should certainly be possible to approach this issue empirically.

With three basic colors there are also three basic binary combinations (Note 38), namely blue-yellow, blue-red and yellow-red. Here blue-yellow (Fig. 47 ) is the most basic, because essentially the cool-warm contrast, most centrally represented as teal-orange (Fig. 13). This is probably the common color contrast (Note 39) in classical Western painting. The other two are less common, but still common enough. Figure 48 shows an extreme example of yellow-red. It may easily be judged 'intrusive,' or even 'irritating.' Blue-red is fairly common (Fig. 49), often rated 'calm,' sometimes 'gloomy,' but even 'irritating.' Indeed, because blue, yellow and red are to be taken in an extended sense, these combinations actually subtend a wide spectrum and may evoke a wide range of affective responses.

Of course, there are also various 'in-between' contrasts. These tend to look 'special' (an example purple-green, Fig. 50), possibly because they fail to fit the framework. It makes them of great interest to certain painters. A research focusing on such cases might well prove to be rewarding.

In retrospect, we cannot claim much of a rigid connection between color combinations and affective responses. Instead, what most usefully results from a study like the present one is an outline of a generic, qualitative structure of the color gamut. We expect variations in procedure to lead to similar conclusions, obviously an expectation that should be followed up. 


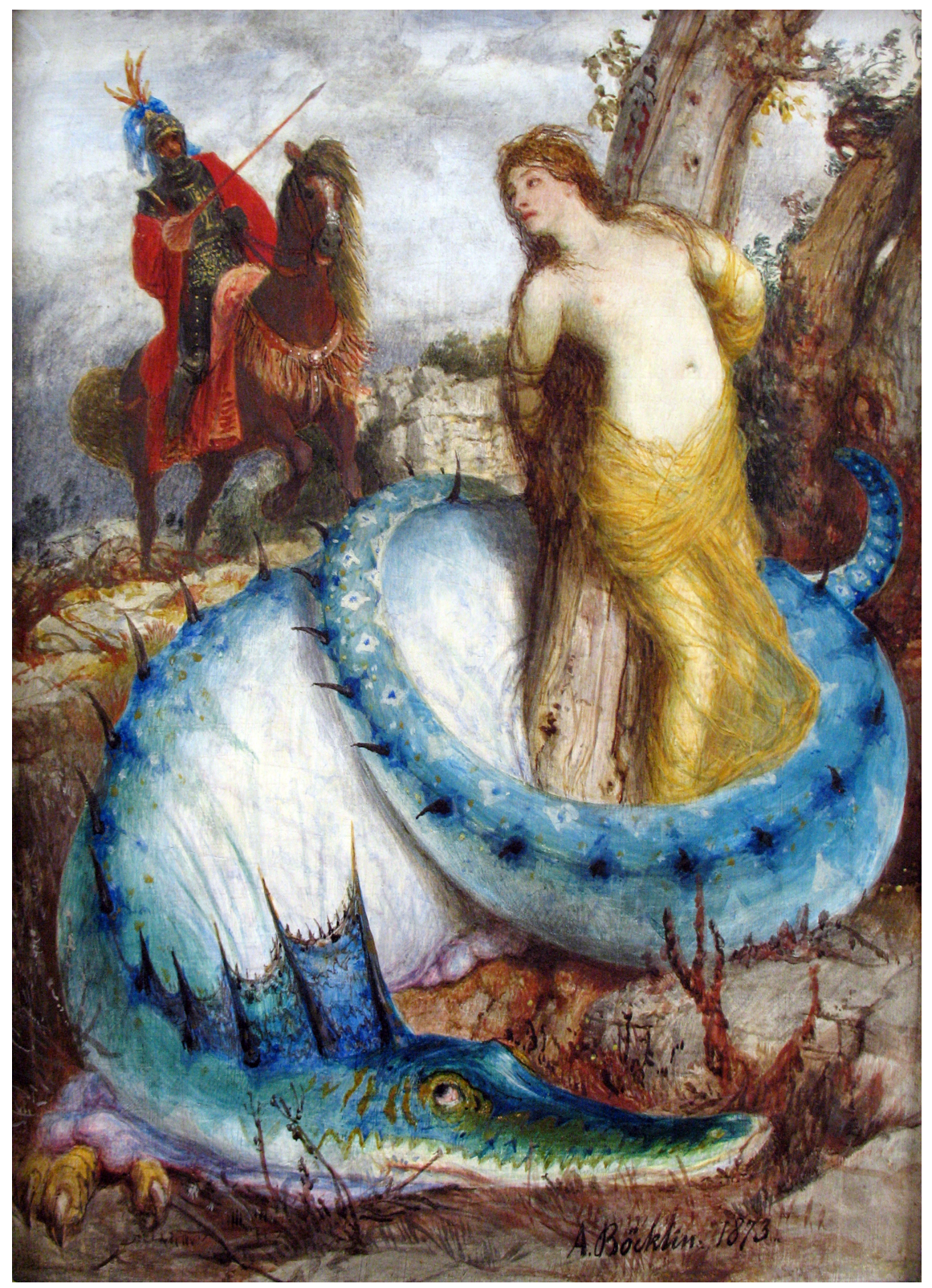

Figure 46. Arnold Böcklin (1827-1901), Ruggiero und Angelica (1873). A rare example of a KWRYC combination, straddling the neutral axis (RY against C). Colors have been assigned to actors, with minor crossovers (e.g, the claws of the beast, the plumage on Ruggiero 's helmet) that are important in the overall composition. This color combination would hardly look good in a texture. 


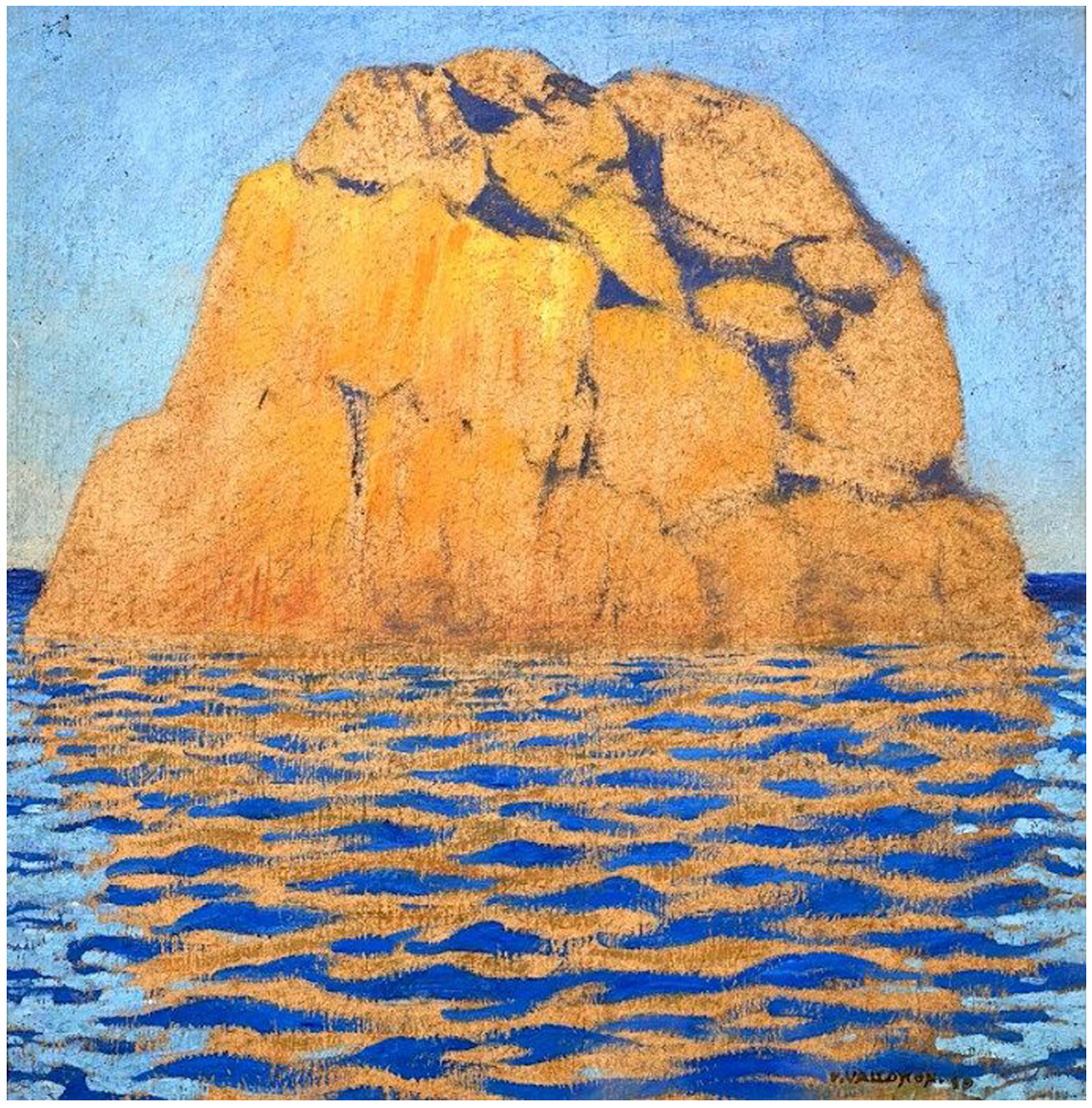

Figure 47. Félix Vallotton (1865-1925), Rocher à Ploumanach (1917). A perfect example of the blue-yellow combination. A very strong pattern. Lively, vivid, irritating, cheerful, intrusive, piercing? Some of these, rather than their opposites. We would probably pick 'vivid,' or 'lively.' How much depends upon the topic? Does it work out the same upside down? Stimuli like those in Fig. 1 hardly address such issues.

\subsection{Spatial Structure}

Our research is singled out because of the stimuli used in the study. Instead of the generic, simple juxtaposition of some areas of flat colors (Fig. 1), these stimuli were actually appreciated by the participants for æsthetic reasons (Fig. 51). Apart from the unconventional spatial structure, the stimuli are unusual in this kind of research because they include the black-white dimension. This is generic in paintings, but rarely used in 'color harmony' (as it tends to be referred to) research. 


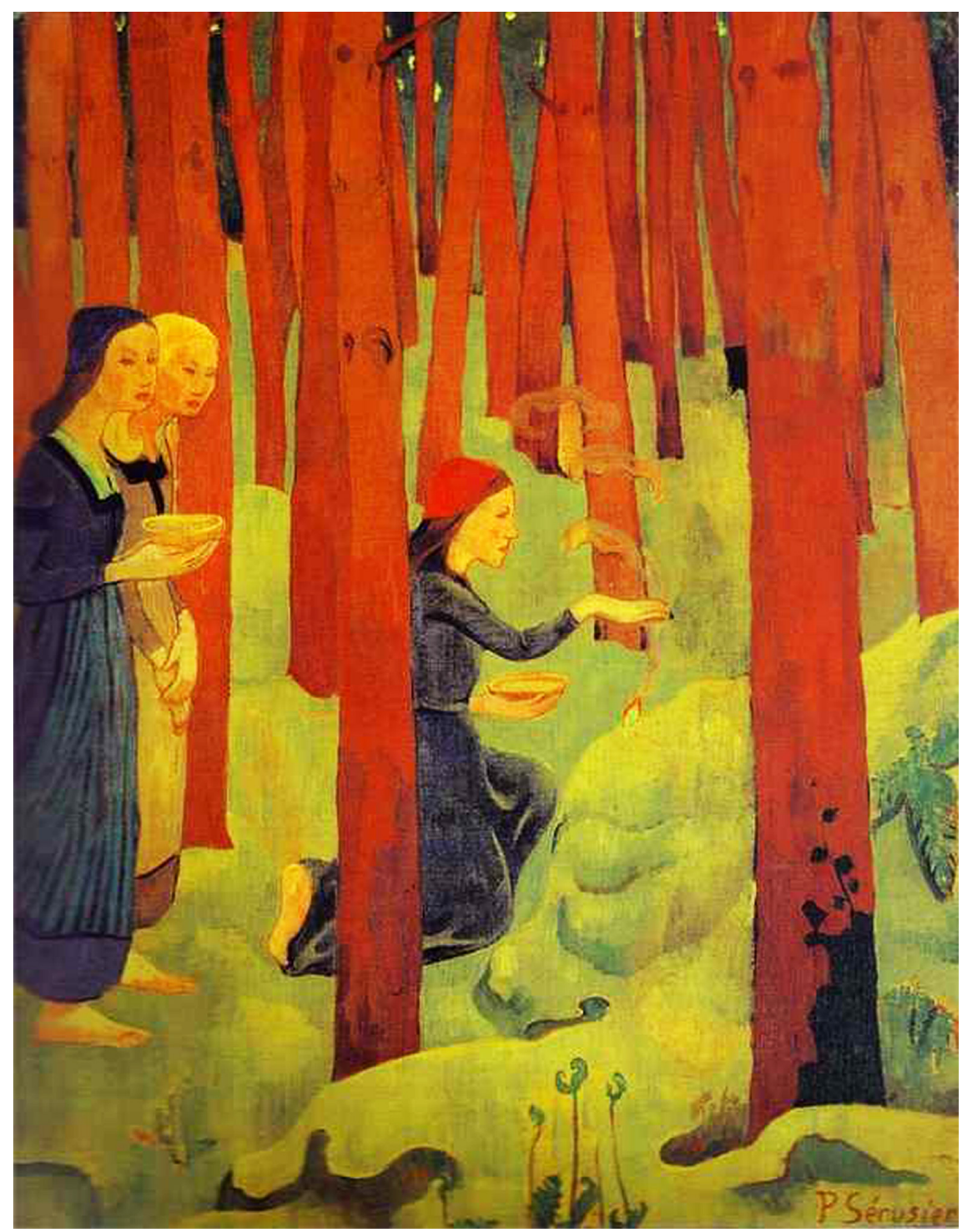

Figure 48. Sérusier, Le Forêt Sacré (1891). An extreme yellow-red combination. What about such a scheme? Perhaps 'piercing and irritating?' Ratings are bound to differ. The nominal subject may influence the choice.

We find little difference in the comparison of the textures and mosaics. Apparently, affective responses were mainly determined by the color combinations. This is perhaps not too surprising. For what to expect? Maybe a bias due to the overall style of spatial structure, hardly something hue-specific. Anyway, we did not find any. 


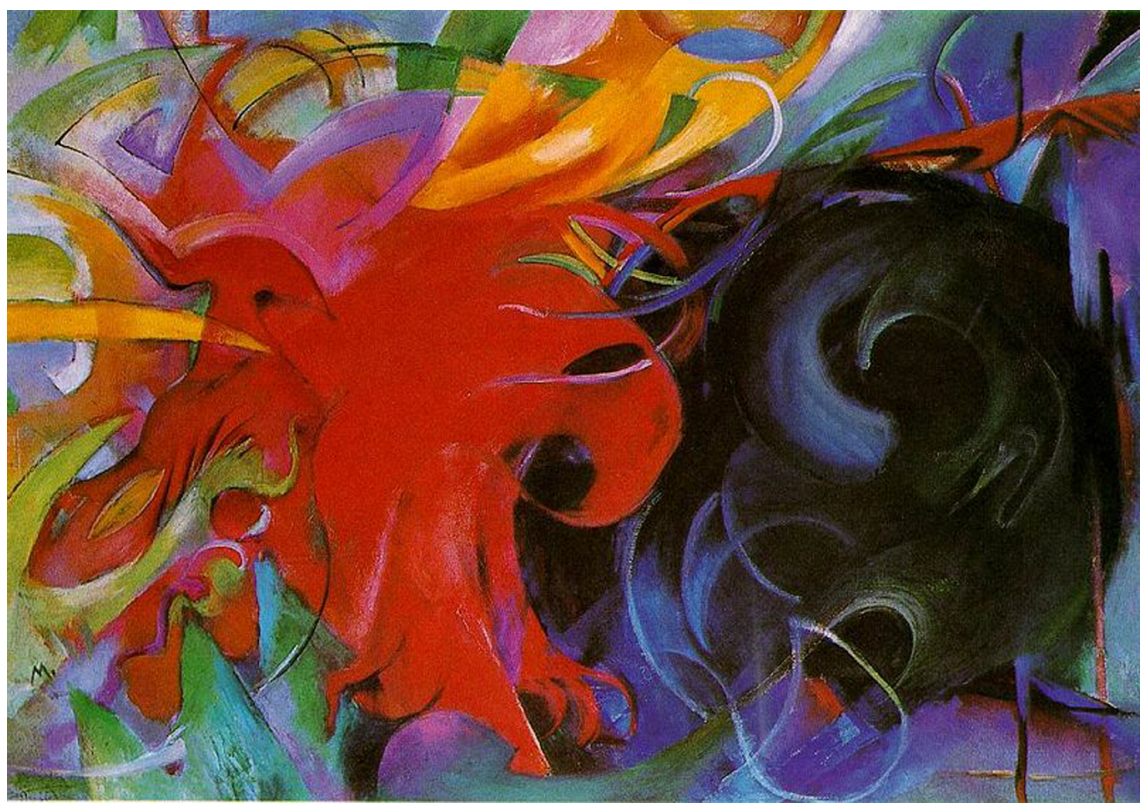

Figure 49. Franz Marc (1880-1916), Kämpfende Formen (1914.). A blue-red combination with a yellow accent. The turmoil of the aggressive shapes changes the affective value of the colors here. How would one rate it? 'Intrusive,' or 'irritating' perhaps? Certainly not 'dull,' but perhaps a touch of 'calm?' Up to the viewer. Knowing the title will likely make a difference. Knowing about Marc will make another.

It is different with the foreground/background patterns. Here we have several examples of the same color combinations having different affective connotations, depending on which of the colors assumes the role of foreground. One might well expect specific differences due to ecological factors (Koenderink et al., 2015). For instance, red typically occurs in the foreground (except at sunset), whereas blues and greens tend to occur in the backgrounds (Note 40).

If this inference applies, then it opens up a generic line of research that might well turn out to be rewarding. We have already some experience with this for the case of the position of certain colors in the image frame (Koenderink et al., 2015). 


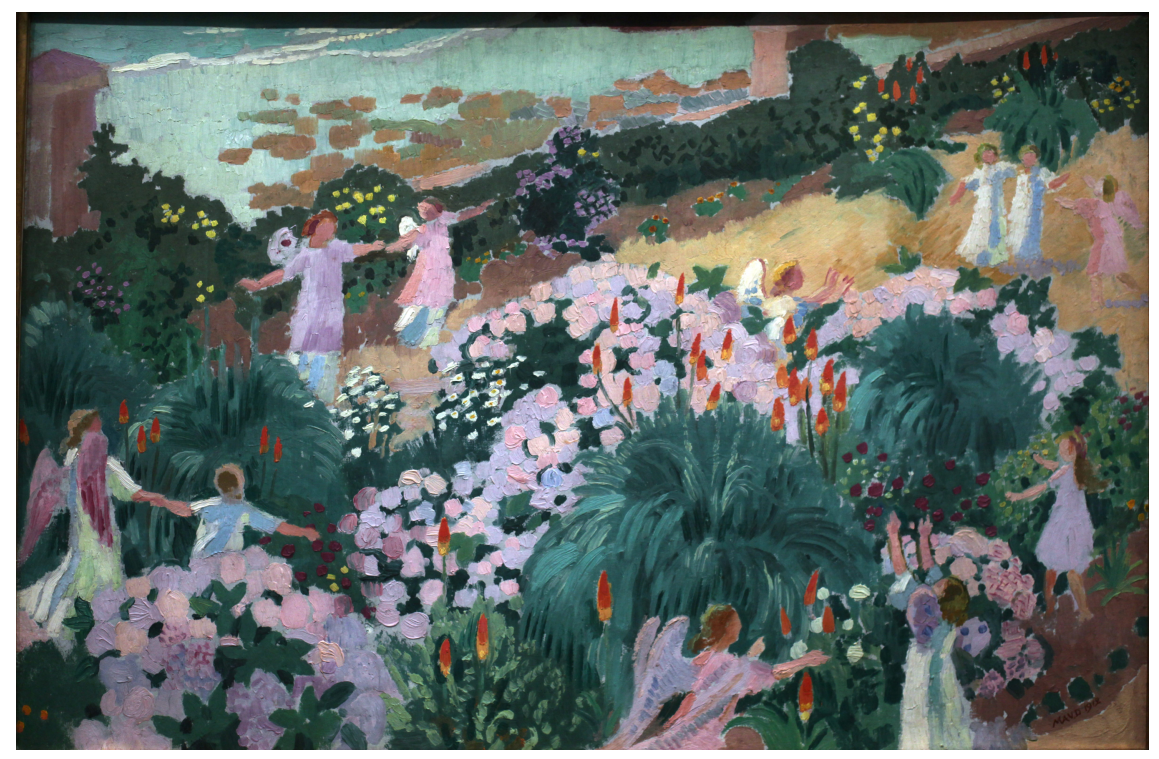

Figure 50. Maurice Denis (1870-1943), Le Paradis (1912). A rare example of a purple (tint)teal (shade) combination. Yellow and pale cyan are extensive, red only punctate accents. These are important in the composition, but not decisive for the color scheme. Together it makes for a perhaps tetrachromatic scheme. How might it be rated? Calm, subdued, or soothing appear to apply, whereas gloomy, piercing, or irritating certainly do not. It seems to us that such ratings are at least not completely arbitrary. They are not very determinate either.

\section{Conclusions}

There are three major 'take home' messages from this research:

- Both color preferences and affective responses throughout the population are not fully idiosyncratic. The (not uncommon) notion that color harmony does not exist because fully idiosyncratic is factually wrong. Instead, responses are to a large part systematic (Fig. 19). Reasons are not likely to be cultural.

- Warm and cool are not just artist's fancies. They pop up as the major dichotomic structure in the appreciation of colored patterns by the generic population (Fig. 23). One does not need to have gone through art school training for that.

- There is an underlying bedrock structure that is based upon blue, yellow and red - taken in an extended sense (Fig. 39). The blue and yellow are the cool-warm bisection of the daylight spectrum. (Teal-orange is actually closer, but just blue-yellow in a wide sense.) Red is an extra structural distinction on top of that. 


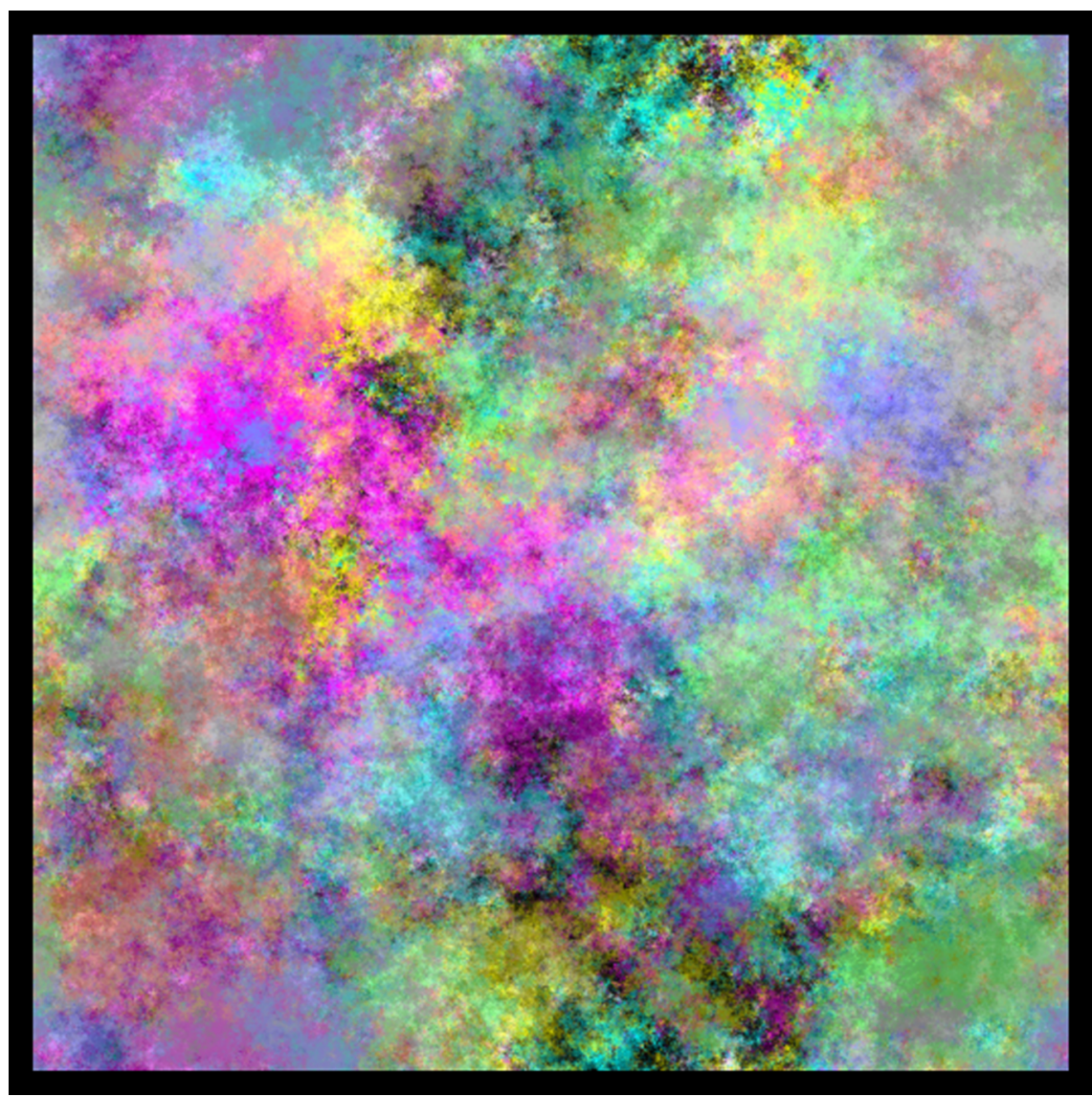

Figure 51. Example stimulus. This image is an equal mixture of black, white, cyan, magenta and yellow. It is completely random, thus yields rich material for the imagination. No participant was ever bored.

Of course, one may further speculate on the basis of any of these items. There are plenty of leads for further academic endeavors. One may also attempt to apply such insights in fact-guided industrial design education, Bauhaus-style, say. Here we leave it at a few remarks.

The first two points are closely related. Given the linguistic data, it is indeed unlikely that the striking similarities between these affective responses are merely cultural. That implicates that they must be ecological/ethological, Homo sapiens's evolutionary heritage from the hominins. This suggests a large agenda of academic issues.

The third point is also related to that, but it suggests problems that might be addressed in experimental phenomenological research. The 'blue' is actually cyan (or you could not use it to mix green). If so, then why is 'true blue' not in 
the same league as red? In terms of the Goethe-Schopenhauer 'parts of white,' cyan is the union of blue and green, whereas yellow is the union of green and red. If blue is split off from cyan, like red from yellow, then purple pops up as a union of blue and red, just as green pops up as the intersection of yellow and cyan. There is a lot of structure to probe here. Somehow, it (by evolutionary processes) ended up in a lopsided manner. There should be a story here.

In retrospect, these findings appear to corroborate the artistic intuitions. The De Stijl/Bauhaus palette is indeed special, not just an arbitrary gimmick. Moreover, it is perfectly tuned to the masses (Note 41), very much in line with the spirit of the time (Note 42).

Anyway, affective color is best understood in terms of blue, yellow and red and is remarkably constant throughout the population. Most likely an ethological fact.

\section{Acknowledgements}

Experiments were run at the Department of Psychology, Justus Liebig University, Giessen, Germany. We are most grateful to our volunteer participants. We thank Karl Gegenfurtner for providing a congenial environment. Jan Koenderink was supported by the Alexander von Humboldt Foundation and gratefully acknowledges the support of the Miller Institute at the University of California at Berkeley. Doris Braun was supported by the Deutsche Forschungsgemeinschaft (DFG, German Research Foundation), project number 222641018- SFB/TRR 135 Project A1. The work was supported by the program by the Flemish Government (METH/14/02), awarded to Professor Johan Wagemans.

\section{Supplementary Material}

Supplementary material is available online at: https://doi.org/10.6084/m9.figshare.16700209

\section{Notes}

1. A wisdom apparently already familiar to the ancient Greeks (third century BCE). Current form said to originate from Margaret Wolfe Hungerford's book 'Molly Bawn' (1878).

2. According to freely offered remarks by the participants in the experiment and by various attendants of VSAC-2019 and ECVP-2019 (van Doorn et al., 2019a, b).

3. The 'Supplementary material' contains mainly material of a technical nature. 
4. See also Douchová (2015) on this issue. It has a clear exposition of Birkhoff's formal proposition and an extensive bibliography. Birkhoff's measure is similar to the notion of Prägnanz used in Gestalt psychology.

5. Just for the record: this informal test did not involve the stimuli used in the actual experiments! Thus, the participants were simply asked to state their preferences, they did not look at any form of display. It thus involves the issue of words versus colored patches (Jonauskaite et al., 2020), as in the comparable experiment of Albertazzi et al. (2015).

6. Remember that each color actually stands as a representative for a rather extensive environment. Thus, 'brown' includes a range from dark oranges to olives and so forth. This should be kept in mind when perusing the figures.

7. There is a huge literature on color preferences (impossible to cite or review here. For a start see Oegema van der Wal, 1963; Crozier, 1999; Dittmar, 2001; Ellis and Ficek, 2001; Teller et al., 2004).

8. Discrimination data suggest up to $4 \times 10^{7}$. More practically oriented methods suggest much smaller values, say about $10^{3}$ (Koenderink et al., 2018).

9. To be precise, $166,666,666,666,500,000$. The number of proper subsets is close to 10301029 . Not infinity by a long shot, but close enough for the rest of us. The point is that one soon gets into large numbers.

10. We do not count the empty set \{\} . We will often use an abridged notation, e.g, refer to the subset of achromatic colors $\{\mathrm{K}, \mathrm{W}\}$ as $\mathrm{KW}$, and so forth.

11. Anders Leonard Zorn (1860-1920) used lead white (flake white), yellow ochre, vermilion and ivory black. (But note that an olive green color can be mixed from ivory black and yellow ochre.)

12. To be understood in the classical figure/ground sense. Thus, figure and ground are phenomenologically defined, not as physical image structures.

13. As happens when independent images are placed in the RGB pixel components. Such images present a cacochromy to the eye.

14. Many artists ignore black, they simply mix complementary colors. Few would ignore white. Only 'pure' water color can do that, using the white paper base. But even many water color artists will have a tube of white ready (technically the water color tends to gouache then). Böcklin freely used both black and white, but was conservative in mixing pure hues.

15. Much like the pages of the Munsell atlas.

16. There is not that much formal literature. However, a query 'teal and orange' (no quotes) on Google search will yield dozens of comments and numerous illustrative images. 
17. Apparent exceptions are often explained by the shifting nature of foreand background. For instance, the directly and indirectly lit parts of a single object are typically used much like fore- and background. Another technique is to bind the hue to pictorial significant objects, essentially the same idea.

18. We skip the topic of achromatic chords here. This is an interesting and important topic in in own right. In artistic usage it remains largely disjunct from chromatic harmonies. In this paper we concentrate mostly on chromatics.

19. Using the centroid for the cluster dissimilarity function.

20. Pearson's $\chi^{2}$ statistics yields a $p$ value of 0.80 . A quantile-quantile (QQ)plot is almost perfectly straight.

21. Here we simply correlate the data by way of the Pearson correlation definition. We have also explored various non-parametric measures of similarity, but this makes essentially no difference for the results. The simple, direct approach then seems indicated.

22. With some effort one might be able to increase the size of the group a little. However, it is probably not worth that effort.

23. Although Delacroix's triangle and Klee's circle are topologically identical, they suggest a slightly different understanding. For instance, Delacroix's triangle appears to be hollow, whereas Klee likely saw the center as white. This evidently requires an extensive study by itself.

24. Courses on color by Johannes Itten, Wassily Kandinsky, Paul Klee and others. These artists had little affinity to the scientific perspective (Ball and Ruben, 2004; Schawelka, 2018).

25. De Stijl remained leading till 1931. Main artists were Theo van Doesburg (who published the journal De Stijl), Piet Mondriaan, Bart van der Leck, Gerrit Rietveld and others. Their famous palette had the primary colors blue, yellow and red as well as the primary values, black, white, and gray. (Gray is indeed a color, not just a neutral between white and black; Nayatani and Sakai, 2014.)

26. Maybe coarse-graining has the generic effect to raise correlations? After all, averaging smoothes over differences, thus may result in increased similarity. This is an issue that needs to be investigated by quantitative means.

27. We have not explicitly looked for patterns (Palmer, 1999; Jameson, 2005). To do so makes sense, at least in principle. However, we are reluctant to mine the data for minor effects.

28. The reader is likely to come to the same conclusion after looking at some instances. It might be of interest to turn this into a formal experiment. A work of art should capture the attention at a cursory glance - only to have more to offer at a good look. 
29. Not too surprising perhaps, because there is a lot of slop.

30. A much used and indeed key concept in analytical philosophy (Crane, 2015). However, the term is almost a contradictio in terminis from a phenomenological perspective (Koenderink, 2019).

31. Much like gray is not a neutral between black and white, but a color category all by itself.

32. Think of taking averages, standard deviations, perform principal components analysis and so forth, when the space is neither linear, nor metric, maybe not even has a well-defined topological structure. It is common enough to see formal methods applied in non-appropriate settings. Without a priori knowledge of the domain it is best to proceed with the simplest methods.

33. Technically, the 'problem of induction.'

34. Note that ' $G$ ' stands for yellow (Gelb), 'Gr' for green (Grün).

35. In 'true' watercolor white is not required, indeed 'white watercolor' is a contradiction in terms. 'Wise' choices in opaque media concern physical and chemical properties.

36. A good 'cool black' is actually a dark shade of cyan. This allows one to obtain greens through mixture with yellows.

37. Here linguistic color terms are monolexemic, monomorphemic, not included in other terms and generic, that is to say, not restricted to a narrow context, and moreover salient.

38. Neither monochromatic, nor trichromatic patterns are generic 'combinations.' Only the dichromatic combinations are proper subgroups.

39. Apart from dark-light! But we are considering chromatic contrast here.

40. Vision science often interprets this as due to '(binocular) chromostereopsis,' but this is actually unlikely. The effect is probably ethological, rather than physiological. Some additional research would be welcome.

41. In the sense that - as we found - the responses of a large group of naive observers are concordant in the Bauhaus style, although there exist significant subgroups if the hues are further differentiated.

42. Thus, Walter Gropius wrote in the Program of the Staatliche Bauhaus in Weimar: "So let us therefore create a new guild of craftsmen, free of the divisive class pretensions that endeavored to raise a prideful barrier between craftsmen and artists! Let us strive for, conceive and create the new building of the future that will unite every discipline, architecture and sculpture and painting, and which will one day rise heavenwards from the million hands of craftsmen as a clear symbol of a new belief to come." (https://bauhausmanifesto.com). 


\section{References}

Albers, J. (1963). Interaction of Colour. Yale University Press, New Haven, CT, USA.

Albertazzi, L. (2013). Experimental phenomenology: an introduction, in: Handbook of Experimental Phenomenology: Visual Perception of Shape, Space and Appearance, L. Albertazzi (ed.), pp. 1-36, John Wiley \& Sons, Ltd, Chichester, UK. doi: 10.1002/978111 8329016.ch.

Albertazzi, L., Koenderink, J. J. and van Doorn, A. (2015). Chromatic dimensions earthy, watery, airy, and fiery, Perception 44, 1153-1178. doi: 10.1177/0301006615594700.

Bakker, I., van der Voordt, T., Vink, P., de Boon, J. and Bazley, C. (2015). Color preferences for different topics in connection to personal characteristics, Color Res. Appl. 40, 62-71. doi: 10.1002/col.21845.

Ball, P. and Ruben, M. (2004). Color theory in science and art: Ostwald and the Bauhaus, Angew. Chem. Int. Ed. 43, 4842-4847. doi: 10.1002/anie.200430086.

Batchelor, D. (2000). Chromophobia. Reaktion Books, London, UK.

Beke, L., Kutas, G., Kwak, Y., Sung, G. Y., Park, D.-S. and Peter Bodrogi, P. (2008). Color preference of aged observers compared to young observers, Color Res. Appl. 33, 381-394. doi: $10.1002 / \mathrm{col} .20434$.

Berlin, B. and Kay, P. (1969). Basic Color Terms: Their Universality and Evolution. University of California Press, Berkeley, CA, USA.

Birkhoff, G. D. (1933) Aesthetic Measure. Harvard University Press, Cambridge MA, USA.

Blanc, C. (1891) The Grammar of Painting and Engraving. S.C. Griggs and Company, Chicago, IL, USA.

Boynton, R. M. (1989). Eleven colors that are almost never confused, Proc. Vol. 1077, Human Vision, Visual Processing, and Digital Display. doi: 10.1117/12.952730.

Burchett, K. E. (2002). Color harmony, Color Res. Appl. 27, 28-31. doi: 10.1002/col.10004.

Carpenter, H., B. (1915). Colour: a Manual of its Theory and Practice. B. T. Batsford, London, UK.

Chen, N., Tanaka, K., Matsuyoshi, D. and Watanabe, K. (2016). Cross preferences for colors and shapes, Color Res. Appl. 41, 188-195. doi: 10.1002/col.21958.

Chevreul, M. E. (1855). Principles of Harmony and Contrast of Colours, and Their Applications to the Arts, 2nd ed. Longman, Brown, Green, and Longmans, London, UK.

Chijiiwa, H. (1987). Color harmony. a guide to creative color combinations. Rockport Publishers, Gloucester, MA, USA.

Clarke, T. and Costall, A. (2008). The emotional connotations of color: A qualitative investigation, Color Res. Appl. 33, 406-410. doi: 10.1002/col.20435

Crane, T. (2015). The problem of perception, in: Stanford Encyclopedia of Philosophy, E. N. Zalta (Ed.), first published Mar. 8, 2005; substantive revision Dec. 31, 2015. Available at https://plato.stanford.edu/entries/perception-problem/.

Crozier, W. R. (1999). The meanings of colour: preferences among hues, Pigment Resin Technol. 28, 6-14. doi: 10.1108/03699429910252315.

Derefeldt, G., Swartling, T., Berggrund, U. and Bodrogi, P. (2004). Cognitive color, Color Res. Appl. 29, 7-19. doi: 10.1002/col.10209. 
Dittmar, M. (2001). Changing colour preferences with ageing: a comparative study on younger and older native Germans aged 19-90 years, Gerontology 47, 219-226. doi: 10.1159/ 000052802 .

Douchová, V. (2015). Birkhoff's aesthetic measure, Acta Univ. Carol. Philos. Hist. 21, 39-53. doi: $10.14712 / 24647055.2016 .8$.

Efron, B. (1981). Nonparametric estimates of standard error: The jackknife, the bootstrap and other methods, Biometrika 68, 589-599. doi: 10.1093/biomet/68.3.589.

Ellis, L. and Ficek, C. (2001). Color preferences according to gender and sexual orientation, Pers. Individ. Dif. 31, 1375-1379. doi: 10.1016/S0191-8869(00)00231-2.

Fechner, G. T. (1866). Das Associationsprincip in der Aesthetik, Z. Bild. Kunst 1, 179-191.

Geymonat de Destefani, L. R. and Whitfield, T. W. A. (2008). Esthetic decision-making: How do people select colours for real settings? Color Res. Appl. 33, 55-60. doi: 10.1002/col. 20361.

Goethe, J. W. (1810). Zur Farbenlehre. Cotta, Tübingen, Germany.

Granger, G. W. (1955). An experimental study of colour harmony, J. Gen. Psychol. 52, 21-35. doi: 10.1080/00221309.1955.9918341.

Granville, W. C. (1987). Color harmony: What is it? Color Res. Appl. 12, 196-201. doi: 10.1002/col.5080120407.

Griffin, L. D. and Mylonas, D. (2019). Categorical color geometry, PLoS ONE 14, e0216296. doi: 10.1371/journal.pone.0216296.

Hanada, M. (2013). Analyses of color emotion for color pairs with independent component analysis and factor analysis, Color Res. Appl. 38, 297-308. doi: 10.1002/col.20750.

Heddell, P. (1988). Color harmony: New applications of existing concepts, Color Res. Appl. 13, 55-57. doi: 10.1002/col.5080130111.

Hethorn, J. (2005). Understanding aesthetic preference: approaches toward improved product development, J. Text. Apparel Technol. Manag. 4, 1-11.

Hume, D. (1910 [orig. 1757]). Of the standard of taste, in: English Essays from Sir Philip Sidney to Macaulay, C. W. Eliott (Ed.), pp. 215-236. P. F. Collier \& Son, New York, NY, USA.

Jameson, K. A. (2005). Why GRUE? An interpoint-distance model analysis of composite color categories, Cross-Cult. Res. 39, 159-204. doi: 10.1177/1069397104273766.

Jonauskaite, D., Parraga, C. A., Quiblier, M. and Mohr, C. (2020). Feeling blue or seeing red? Similar patterns of emotion associations with colour patches and colour terms, $i$-Perception 11, 2041669520902484. doi: 10.1177/2041669520902484.

Kay, P. and Maffi, L. (1999). Color appearance and the emergence and evolution of basic color lexicons, Am. Anthropol. 101, 743-760. doi: 10.1525/aa.1999.101.4.743.

Kim, Y.-J. (2013). Can eyes smell? Cross-modal correspondences between color hue-tone and fragrance family, Color Res. Appl. 38, 139-156. doi: 10.1002/col.20717.

Kobayashi, S. (2010). Color Image Scale, Kodansha International, Tokyo, Japan.

Koenderink, J. (2010). Colour for the Sciences. MIT Press, Cambridge MA, USA.

Koenderink, J. J. (2019). Sentience, De Clootcrans Press, Trajectum, The Netherlands. Available at http://www.gestaltrevision.be/en/resources/clootcrans-press.

Koenderink, J., Doorn, A. van, Albertazzi, A. and Wagemans, J. (2015). Hue contrast and the sense of space, i-Perception 6, 67-85. doi: 10.1068/i0701. 
Koenderink, J. and van Doorn, A. (2021). Orange \& Teal, Art Percept. 9, 134-166. doi: 10.1163/22134913-bja10018.

Koenderink, J., van Doorn, A. and Gegenfurtner, K. (2018). Graininess of RGB-display space, i-Perception 9, 1-46. doi: 10.1177/2041669518803971.

Likert, R. (1932). A technique for the measurement of attitudes, Arch. Psychol. 140, 1-55.

Maxwell, J. C. (1857). Experiments on colour, as perceived by the eye, with remarks on colour blindness, Trans. R. Soc. Edinburgh 21, 275-289. doi:10.1017/S0080456800032117.

Moon, P. and Spencer, D. E. (1944a). Geometric formulation of classical color harmony, J. Opt. Soc. Am. 34, 46-59. doi: 10.1364/JOSA.34.000046.

Moon, P. and Spencer, D. E. (1944b). Area in color harmony, J. Opt. Soc. Am. 34, 93-103. doi: 10.1364/JOSA.34.000093.

Moon, P. and Spencer, D. E. (1944c). Aesthetic measure applied to color harmony, J. Opt. Soc. Am. 34, 234-242. doi: 10.1364/JOSA.34.000234.

Nayatani, Y. and Sakai, H. (2014). Gray and grayness - its complexities in color appearance of surface colors, Color Res. Appl., 39, 37-44. doi: 10.1002/col.21758.

Newton, I. (1704). Opticks: or, A Treatise of the Reflexions, Refractions, Inflexions and Colours of Light. Sam. Smith and Benj. Walford, London, UK.

Nietzsche, F. (1988 [orig. 1873]). Über Wahrheit und Lüge im aussermoralischen Sinne, in: Ecce Homo: How One Becomes What One is. Sämtliche Werke: Kritische Studienausgabe in 15 Bänden, G. Colli and M. Montinari (Eds), p. 875. Walter de Gruyter, Berlin.

Oegema van der Wal, Th. (1963). Proeve ener Theoretische Kleurenspychologie. WereldBibliotheek, Amsterdam.

Ortlieb, S. A., Kügel, W. A. and Carbon, C.-C. (2020). Fechner (1866): The aesthetic association principle — a commented translation, i-Perception 11. doi: 10.1177/2041669520920309.

Ostwald, W. (1917a). Die Farbenfibel. Unesma, Leipzig, Germany.

Ostwald, W. (1917b). Der Farbatlas. Unesma, Leipzig, Germany.

Ostwald, W. (1918). Die Harmonie der Farben. Unesma, Leipzig, Germany.

Ostwald, W. (1919). Einführung in die Farbenlehre. Unesma, Leipzig, Germany.

Ostwald, W. (1922). Die Harmonie der Formen. Unesma, Leipzig, Germany.

Overy, P. (1991). De Stijl (World of Art). Thames and Hudson Ltd, London, UK.

Palmer, S. E. (1999). Color, consciousness, and the isomorphism constraint, Behav. Brain Sci. 22, 923-989. doi: 10.1017/S0140525X99002216.

Palmer, S. E., Schloss, K. B. (2010). An ecological valence theory of human color preference. Proc. Natl Acad. Sci. U. S. A. 107, 8877-8882. doi: 10.1073/pnas.0906172107.

Pantone (2020). https://www.pantone.com. Retrieved 07-02-2020.

Pawlik, J. (1969). Theorie der Farbe. Eine Einführung in begriffliche Gebiete der ästhetischen Farbenlehre. Dumont Schauberg, Köln, Germany.

Pope, A. (1944). Notes on the problem of color harmony and the geometry of color space with reference to articles by Moon and Spencer, J. Opt. Soc. Am. 34, 759-765. doi: 10.1364/ JOSA.34.000759.

Popper, K. (2002). Conjectures and Refutations. Routledge, New York, NY, USA.

Quiller, S. (1989). Color Choices: Making Color Sense Out of Color Theory. Watson-Guptill, New York, NY, USA. 
Richards, W. A. (1987). Introduction, in: Image Understanding 1985-86, W. Richards and S. Ullman (Eds), pp. v-xvii. Ablex, Norwood, NJ, USA.

Rijgersberg, E. (1938). Beknopte Kleurenleer en de Toepassing van de Kleuren in Architectuur en Binnenhuiskunst. Argus, Amsterdam, The Netherlands.

Runge, P. O. (1810). Die Farben-Kugel, oder Construction des Verhältnisses aller Farben zueinander. Perthes, Hamburg, Germany.

Schawelka, K. (2018). Wilhelm Ostwald's "Harmony of Colours" (1918) and its mixed reception - a reassessment, Óbuda Univ. e-Bull. 8, 13-24.

Schloss, K. B., Strauss, E. D. and Palmer, S. E. (2013). Object color preferences, Color Res. Appl. 38, 393-411. doi: 10.1002/col.21756.

Schloss, K. B, Witzel, C. and Lai, L. Y. (2020). Blue hues don't bring the blues: questioning conventional notions of color-emotion associations, J. Opt. Soc. Am. A 37, 813-824. doi: 10.1364/JOSAA.383588.

Schopenhauer, A. (1816). Ueber das Sehn und die Farben: eine Abhandlung, 2nd ed. Hartknoch, Leipzig, Germany.

Skinner, B. F. (1938). The Behavior of Organisms: An Experimental Analysis. AppletonCentury, New York, NY, USA.

Smith, D. (2008). Color-Person-Environment Relationships, Color Res. Appl. 33, 312-319.

Solli, M. and Lenz, R. (2011). Color emotions for multi-colored images, Color Res. Appl. 36, 210-221. doi: 10.1002/col.20604.

Son, J., Park, J., Kang, D., Belal, S. A., Cha, J. and Shim, K. (2020). Effects of white, yellow, and blue colored LEDs on milk production, milk composition, and physiological responses in dairy cattle, Anim. Sci. J. 91, e13337. doi: 10.1111/asj.13337.

Stone, E. (2004). The Dynamics of Fashion, 2nd ed. Fairchild Publications, New York, NY, USA.

Tangkijviwat, U., Rattanakasamsuk, K. and Shinoda, H. (2010). Color preference affected by mode of color appearance, Color Res. Appl. 35, 50-61. doi: 10.1002/col.20536.

Teller, D. Y., Civan, A. and Bronson-Castain, K. (2004). Infants' spontaneous color preferences are not due to adult-like brightness variations, Vis. Neurosci. 21, 397-401. doi:10.1017/ S0952523804213360.

Tukey, J. W. (1977) Exploratory Data Analysis. Addison Wesley, Reading, MA, USA.

van Doorn, A., Braun, D. and Koenderink, J. (2019a). Affective reactions to coloured patterns, in: 42nd European Conference on Visual Perception (ECVP) 2019 Leuven, Belgium. Perception 48, 193.

van Doorn, A., Braun, D. and Koenderink, J. (2019b). Foray into colour harmony, in: VSAC 2019 7th Visual Science of Art Conference 21-24 August 2019, Leuven, Belgium, p. 15. Available at https://www.vsac2019.org/pdf/Abstractbook_20190808.pdf.

von Bezold, W. (1874). Die Farbenlehre in Hinblick auf Kunst und Kunstgewerbe. George Westermann, Braunschweig, Germany.

von Helmholtz, H. (1855). Ueber die Zusammensetzung von Spectralfarben, Ann. Phys. Chem. 94, 1-28. doi: 10.1002/andp.18551700102.

Wertheimer, M. (1924). Gestalt theory, in W. D. Ellis (Ed.) (1938), A Source Book of Gestalt Psychology, Harcourt Brace, New York, NY, USA. Available at http://gestalttheory.net/ archive. 
Yarkoni, T. (2020). The generalizability crisis. Behav. Brain Sci. 1-37. doi: 10.1017/ S0140525X20001685.

Zangwill, N. (2003/2019). Aesthetic judgment, in: Stanford Encyclopedia of Philosophy, E. N. Zalta (Ed.), first published Feb. 28, 2003; substantive revision Jan. 28, 2019. Available at https://plato.stanford.edu/entries/aesthetic-judgment/; retrieved 20-01-2021. 